\title{
LOCAL THETA CORRESPONDENCES BETWEEN EPIPELAGIC SUPERCUSPIDAL REPRESENTATIONS
}

\author{
HUNG YEAN LOKE, JIA-JUN MA, AND GORDAN SAVIN
}

\begin{abstract}
In this paper we study the local theta correspondences between epipelagic supercupsidal representations of a type I classical dual pair $\left(G, G^{\prime}\right)$ over $p$-adic fields. We show that, besides an exceptional case, an epipelagic supercupsidal representation $\pi$ of $\widetilde{G}$ lifts to an epipelagic supercupsidal representation $\pi^{\prime}$ of $\widetilde{G}^{\prime}$ if and only if the epipelagic data of $\pi$ and $\pi^{\prime}$ are related by the moment maps.
\end{abstract}

\section{INTRODUCTION}

1.1. Let $k$ be $p$-adic field with ring of integers $\mathfrak{o}_{k}$, prime ideal $\mathfrak{p}_{k}=(\varpi)$ and residue field $\mathfrak{f}$ of odd characteristic $p$ where $\varpi$ is a fixed uniformizer. Let $\bar{k}$ be its algebraic closure and let $k^{\text {ur }}$ be the maximal unramified extension of $k$. Let $\nu: \bar{k} \rightarrow Q \cup\{\infty\}$ denote the valuation map where $\nu(\varpi)=1$. We will denote an algebraic variety by a boldface letter, say $\mathbf{H}$. For an algebraic extension $E$ of $k$, we will denote its $E$-points by $\mathbf{H}(E)$, and its $k$ points by the corresponding normal letter $H$.

If $\mathbf{G}$ is an algebraic group, then we let $\mathfrak{g}$ be its Lie algebra or the $k$ points of the Lie algebra, depending on the context. If $G=\mathbf{G}(k)$ acts on a set $X$, then ${ }^{g} x$ will denote $g \cdot x$ for $g \in G$ and $x \in X$. In order to simplify the situation, we always assume that $p$ is sufficiently large compared to the rank of $G$. For an reductive algebraic group, we will fix a maximally $k$-split torus $\mathbf{S}$, a maximally $k^{\mathrm{ur}}$-split torus $\mathbf{T}$ which is defined over $k$ and contains $\mathbf{S}$. Since $\mathbf{G}\left(k^{\mathrm{ur}}\right)$ is always quasi-split, we set $\mathbf{Y}:=\mathrm{Z}_{\mathbf{G}}(\mathbf{T})$ to be the fixed Cartan subgroup of $\mathbf{G}$

1.2. We recall the classification of irreducible type I reductive dual pairs. Let $D$ be a division algebra over $k$ with a fixed involution $\tau$, which is either (i) $k$, (ii) a quadratic field extension of $k$, or (iii) the quaternion algebra over $k$. We continue to use $\nu$ to denote the unique extension of the valuation $\nu$ from $k$ to $D$. Let $\mathfrak{o}_{D}$ be the ring of integers of $D$. Let $V$ be a right $D$-module with an $\epsilon$-Hermitian sesquilinear form $\langle,\rangle_{V}$ and $G=\mathbf{G}(k)=\mathrm{U}\left(V,\langle,\rangle_{V}\right)$ be the unitary group preserving $\langle,\rangle_{V}$. Similar notation applies to $G^{\prime}$ with $\epsilon^{\prime}=-\epsilon$. The $k$-vector space $W=V \otimes_{D} V^{\prime}$ has a natural symplectic form and $\left(G, G^{\prime}\right)$ is an irreducible type I reductive dual pair in $\operatorname{Sp}:=\operatorname{Sp}(W)$.

We will let $\widetilde{E}$ be the inverse image of a subgroup $E$ in the metaplectic $\mathrm{C}^{\times}$-cover $\mathrm{Mp}$ of Sp. We fix a non-trivial additive character $\psi: k \rightarrow \mathrm{C}^{\times}$with conductor $\mathfrak{p}_{k}$ and consider local theta correspondence $\theta$ arising from the oscillator representation of $\mathrm{Mp}$ with respect to the character $\psi$. For general information on theta correspondences, see [6, 10, 27]. In this paper, we will investigate the theta correspondence between epipelagic supercuspidal representations of $\widetilde{G}$ and $\widetilde{G}^{\prime}$. In many situations, the roles of $G$ and $G^{\prime}$ are interchangeable. In such cases, we only discuss $G$ and extend all objects and notation to $G^{\prime}$ implicitly by adding 'primes'.

1991 Mathematics Subject Classification. 22E46, 22E47.

Key words and phrases. local theta correspondence, stable vector, moment map, epipelagic supercuspidal representation. 
1.3. We briefly review some facts about epipelagic supercuspidal representations. See Section 2 and 23 for more details. Let $\mathcal{B}(\mathbf{G}, k)$ be the (extended) building of $G$. In the setting of type I dual pairs, $G$ and $G^{\prime}$ have compact centers. Therefore $\mathrm{Z}(G) \subseteq G_{x}$ and $G_{x}=G_{[x]}$ for any $x \in \mathcal{B}(\mathbf{G}, k)$ where $[x]$ is the image of $x$ in the reduced building. Following Reeder-Yu [23], we construct a tamely ramified irreducible supercuspidal representation $\pi_{\Sigma}$ of $\widetilde{G}$ in Section 3.4 from the data $\Sigma=(x, \lambda, \chi)$ where

(SD1) $x \in \mathcal{B}(\mathbf{G}, k)$ is an epipelagic point of order $m$ where $p \nmid m$ (c.f. Definition 2.2.1),

(SD2) $\lambda$ is a stable vector in $\mathfrak{g}_{x,-\frac{1}{m}} / \mathfrak{g}_{x,-\frac{1}{m}}+$ and

(SD3) $\chi$ is a character of the stabilizer $\mathrm{S}_{\lambda}$ of $\lambda$ in $G_{x} / G_{x, 0^{+}}$.

We call $\Sigma=(x, \lambda, \chi)$ an epipelagic data of order $m$ for $G$. The supercuspidal representation $\pi_{\Sigma}$ has depth $\frac{1}{m}$ and it is called an epipelagic supercuspidal representation of $\widetilde{G}$. Since the cover $\widetilde{G}$ depends on the dual pair $\left(G, G^{\prime}\right)$, the notation $\pi_{\Sigma}$ only makes sense relative to the dual pair $\left(G, G^{\prime}\right)$.

1.4. Let $\Sigma=(x, \lambda, \chi)$ and $\Sigma^{\prime}=\left(x^{\prime}, \lambda^{\prime}, \chi^{\prime}\right)$ be epipelagic data of $G$ and $G^{\prime}$ of order $m$ and $m^{\prime}$ respectively. Suppose $\theta\left(\pi_{\Sigma}\right)=\pi_{\Sigma^{\prime}}^{\prime}$. By [21,22], $\pi_{\Sigma}$ and $\pi_{\Sigma^{\prime}}^{\prime}$ have the same depth $\frac{1}{m}=\frac{1}{m^{\prime}}$, i.e. $m=m^{\prime}$. It turns out that the data $(x, \lambda, \chi)$ and $\left(x^{\prime}, \lambda^{\prime}, \chi^{\prime}\right)$ are related by a geometric picture which we now briefly explain.

The points $x$ and $x^{\prime}$ correspond to self dual $\mathfrak{o}_{D^{-}}$-lattice functions $\mathscr{L}$ and $\mathscr{L}^{\prime}$ in $V$ and $V^{\prime}$ respectively (c.f. [3, $5,6,[14]$ ). The tensor product $\mathscr{B}=\mathscr{L} \otimes \mathscr{L}^{\prime}$ is a self dual $\mathfrak{o}_{k}$-lattice function in $W$. The quotient $X=\mathscr{B}_{-\frac{1}{2 m}} / \mathscr{B}_{-\frac{1}{2 m}}+$ is an $\mathfrak{f}$-vector space. In (8) we define the moment maps $\mathrm{M}_{-\frac{1}{2 m}}$ and $\mathrm{M}_{-\frac{1}{2 m}}^{\prime}$ :

$$
\mathfrak{g}_{x,-\frac{1}{m}} \stackrel{\mathrm{M}_{-\frac{1}{2 m}}}{\longleftarrow} \stackrel{\mathrm{M}_{-\frac{1}{2 m}}^{\prime}}{\longrightarrow} \mathfrak{g}_{x^{\prime},-\frac{1}{m}}^{\prime} .
$$

Our first result is a refinement of special cases of [22].

Proposition 1.4.1 (Proposition 7.1.1). Suppose $\theta\left(\pi_{\Sigma}\right)=\pi_{\Sigma^{\prime}}^{\prime}$. Then

$$
\text { there exists a } \bar{w} \in \mathrm{X} \text { such that } \lambda=\mathrm{M}_{-\frac{1}{2 m}}(\bar{w}) \text { and } \lambda^{\prime}=-\mathrm{M}_{-\frac{1}{2 m}}^{\prime}(\bar{w})
$$

Condition (M) imposes severe restriction to the ranks of $G$ and $G^{\prime}$ :

Proposition 1.4.2 (Proposition 7.3.2). Suppose $\theta\left(\pi_{\Sigma}\right)=\pi_{\Sigma^{\prime}}^{\prime}$. Then $\left(\mathbf{G}, \mathbf{G}^{\prime}\right)$ or $\left(\mathbf{G}^{\prime}, \mathbf{G}\right)$ is one of the following types:

(i) $\left(\mathrm{D}_{n}, \mathrm{C}_{n}\right)$, (ii) $\left(\mathrm{C}_{n}, \mathrm{D}_{n+1}\right)$, (iii) $\left(\mathrm{C}_{n}, \mathrm{~B}_{n}\right)$, (iv) $\left(\mathrm{A}_{n}, \mathrm{~A}_{n}\right)$ or (v) $\left(\mathrm{A}_{n}, \mathrm{~A}_{n+1}\right)$.

Let $(x, \lambda)$ and $\left(x^{\prime}, \lambda^{\prime}\right)$ be parts of data for $G$ and $G^{\prime}$ of order $m$ respectively satisfying (SD1) and (SD2). It turns out that Condition (M), in all but one exceptional case (see Case (E) in Section 8), is a sufficient condition for the epipelagic supercuspidal representation $\pi_{\Sigma}$ to lift to an epipelagic supercuspidal representation of $\widetilde{G}^{\prime}$. For the ease of explaining in this introduction, we will omit the exceptional case. Using Condition (M), we will construct a group homomorphism $\alpha: \mathrm{S}_{\lambda^{\prime}}^{\prime} \rightarrow \mathrm{S}_{\lambda}$ in Lemma 8.1.1. We can now state a part of the main 8.1.2.

Theorem 1.4.3. Let $\left(G, G^{\prime}\right)$ be an irreducible type I reductive dual pair such that $\left(\mathbf{G}, \mathbf{G}^{\prime}\right)$ has the form (i)-(v) in Proposition 1.4.2. Let $(x, \lambda)$ and $\left(x^{\prime}, \lambda^{\prime}\right)$ be data of $G$ and $G^{\prime}$ respectively of order $m$ satisfying (SD1), (SD2). We assume that we are not in the exceptional Case (E)

(i) Suppose Condition (M) is satisfied. Then for every character $\chi$ of $\mathrm{S}_{\lambda}$,

$$
\theta\left(\pi_{\Sigma}\right)=\pi_{\Sigma^{\prime}}^{\prime}
$$


where $\Sigma=(x, \lambda, \chi), \Sigma^{\prime}=\left(x^{\prime}, \lambda^{\prime}, \chi^{*} \circ \alpha\right)$ and $\chi^{*}$ is the contragredient of $\chi$. In particular the theta lift is nonzero.

(ii) Conversely, suppose $\theta\left(\pi_{\Sigma}\right)=\pi_{\Sigma^{\prime}}^{\prime}$ where $\Sigma=(x, \lambda, \chi)$ and $\Sigma^{\prime}=\left(x^{\prime}, \lambda^{\prime}, \chi^{\prime}\right)$ are epipelagic data. Then

(a) Condition (M) is satisfied so that $\alpha: \mathrm{S}_{\lambda^{\prime}}^{\prime} \rightarrow \mathrm{S}_{\lambda}$ is well-defined and

(b) $\chi^{\prime}=\chi^{*} \circ \alpha$.

8.1.2 also contains a result for the exceptional Case (E) where $\pi_{\Sigma}$ lifts only for half of the characters of $\mathrm{S}_{\lambda}$. This should be compared with [18].

Acknowledgment. We would like to thank Wee Teck Gan and Jiu-Kang Yu for their valuable comments. Hung Yean Loke is supported by a MOE-NUS AcRF Tier 1 grant R-146-000-208-112. Jia-Jun Ma is partially supported by ISF Grant 1138/10 during his postdoctoral Fellowship at Ben Gurion University and HKRGC Grant CUHK 405213 during his postdoctoral fellowship in IMS of CUHK. Gordan Savin is supported by an NSF grant DMS-1359774.

\section{EPIPELAGIC REPRESENTATIONS}

In this section we review Reeder-Yu's construction of epipelagic supercuspidal representations (c.f. [23]).

2.1. Let $k$ be a $p$-adic field as in Section 1.1. Let $k^{\text {ur }}$ be the maximal unramified extension of $k$ with residue field $\overline{\mathfrak{f}}$. We let Fr denote the Frobenius element such that $\operatorname{Gal}\left(k^{\mathrm{ur}} / k\right)=$ $\operatorname{Gal}(\overline{\mathfrak{f}} / \mathfrak{f})=\langle\mathrm{Fr}\rangle$.

2.2. Epipelagic points. Let $\mathbf{G}$ be an algebraic group defined over $k$. Let $E$ be a tamely ramified extension of $k$. For $x \in \mathcal{B}(\mathbf{G}, k) \subseteq \mathcal{B}(\mathbf{G}, E)$, we set up some notation which will be used in the rest of the paper. Let

- $\mathbf{G}(E)_{x, r}(r \geq 0)$ and $\mathfrak{g}(E)_{x, r}$ be the Moy-Prasad filtrations corresponding to $x$.

- $\mathbf{G}(E)_{x}=\operatorname{Stab}_{\mathbf{G}(E)}(x)$ and $\mathbf{G}(E)_{[x]}=\operatorname{Stab}_{\mathbf{G}(E)}([x])$ where $[x]$ is the image of $x$ in the reduced building;

- $\mathrm{G}_{x}(E)=\mathbf{G}(E)_{x} / \mathbf{G}(E)_{x, 0^{+}}$;

- $\mathbf{G}_{x, r}(E)=\mathbf{G}(E)_{x, r: r^{+}}:=\mathbf{G}(E)_{x, r} / \mathbf{G}(E)_{x, r^{+}}$, and

- $\mathrm{g}_{x, r}(E)=\mathfrak{g}(E)_{x, r: r^{+}}:=\mathfrak{g}(E)_{x, r} / \mathfrak{g}(E)_{x, r^{+}}$.

In order to abbreviate the notations of objects corresponding to $G=\mathbf{G}(k)$, we let

- $G_{x, r}:=\mathbf{G}(k)_{x, r}=\mathbf{G}\left(k^{\mathrm{ur}}\right)_{x, r} \cap \mathbf{G}(k)$,

- $\mathfrak{g}_{x, r}:=\mathfrak{g}(k)_{x, r}=\mathfrak{g}\left(k^{\mathrm{ur}}\right)_{x, r} \cap \mathfrak{g}(k)$,

- $G_{x, r: r^{\prime}}:=\mathbf{G}(k)_{x, r} / \mathbf{G}(k)_{x, r^{\prime}}$ and $\mathfrak{g}_{x, r: r^{\prime}}:=\mathfrak{g}(k)_{x, r} / \mathfrak{g}(k)_{x, r^{\prime}}$ for $r<r^{\prime}$.

In order to abbreviate the notations of objects corresponding to $\mathbf{G}\left(k^{\text {ur }}\right)$, we let

- $\mathrm{G}_{x}:=\mathrm{G}_{x}\left(k^{\mathrm{ur}}\right), \mathrm{G}_{x, r}:=\mathrm{G}_{x, r}\left(k^{\mathrm{ur}}\right)$ and $\mathrm{g}_{x, r}:=\mathrm{g}_{x, r}\left(k^{\mathrm{ur}}\right)$.

The quotient space $\mathrm{g}_{x, r}$ is an $\overline{\mathfrak{f}}$-vector space and we denote its dual space $\operatorname{Hom}_{\overline{\mathfrak{f}}}\left(\mathrm{g}_{x, r}, \overline{\mathfrak{f}}\right)$ by $\check{\mathrm{g}}_{x, r}$. We have assumed in the introduction that $p$ is large compared to the rank of $G$. Then by [2, Prop. 4.1], $\check{\mathrm{g}}_{x, r}$ could be identified with $\mathrm{g}_{x,-r}$ via an invariant bilinear form on $\mathfrak{g}$. Since we are only treating classical groups and $p \neq 2$, we will use a trace form defined later in Definition 6.1.1 in this paper. The group $\mathrm{G}_{x}$ acts on $\breve{\mathrm{g}}_{x, r}$. A vector $\lambda \in \breve{\mathrm{g}}_{x, r}$ is called a stable vector if the $\mathrm{G}_{x}$-orbit of $\lambda$ is Zariski closed in $\check{\mathrm{g}}_{x, r}$ and the stabilizer of $\lambda$ in $\mathbf{G}_{x}$ modulo $\mathrm{Z}\left(\mathbf{G}\left(k^{\mathrm{ur}}\right)\right)_{0: 0^{+}}$is a finite group.

Let $\Psi_{k \text { ur }}$ be the set of affine $\mathbf{T}\left(k^{\text {ur }}\right)$-roots. Suppose $x \in \mathcal{A}(\mathbf{S}, k)=\mathcal{A}\left(\mathbf{T}, k^{\mathrm{ur}}\right)^{\mathrm{Fr}}$, i.e. $x$ is in the apartment defined by $\mathbf{S}$. Let $r(x)$ be the smallest positive value in $\left\{\psi(x) \mid \psi \in \Psi_{k^{\text {ur }}}\right\}$. Then $\mathbf{G}\left(k^{\mathrm{ur}}\right)_{x, 0^{+}}=\mathbf{G}\left(k^{\mathrm{ur}}\right)_{x, r(x)}$. 
Definition 2.2.1. Let $m$ be an integer where $p \nmid m$. A point $x$ in the apartment $\mathcal{A}(\mathbf{S}, k)$ is called an epipelagic point of order $m$ if $r(x)=\frac{1}{m}$ and $\breve{\mathrm{g}}_{x, \frac{1}{m}}$ contains a stable vector.

By [9], $m$ is an even integer except when $\mathbf{G}\left(k^{\text {ur }}\right)$ is split and of type $\mathrm{A}_{m-1}$. In particular $m \geq 2$.

2.3. Epipelagic supercuspidal representations. Let $x$ be an epipelagic point of order $m$ so that $r(x)=\frac{1}{m}$. We fix an isomorphism of abelian groups $c: G_{x, r(x): r(x)^{+}} \rightarrow \mathfrak{g}_{x, r(x): r(x)^{+}}$. For the classical group, we choose the isomorphism to be the one induced by the Cayley transform $c(g)=2(g-1)(g+1)^{-1}$ so that $g-1 \equiv c(g)\left(\bmod \mathfrak{g l}(V)_{x, r(x)^{+}}\right)$.

Let $\lambda \in \check{\mathrm{g}}_{x, \frac{1}{m}}^{\mathrm{Fr}}$ be an $\mathfrak{f}$-rational functional on $\mathrm{g}_{x, \frac{1}{m}}$. Then we get a character

$$
\psi_{\lambda}:=\psi \circ \lambda: G_{x, \frac{1}{m}: \frac{1}{m}}{ }^{+}=\mathrm{g}_{x, \frac{1}{m}}(k) \cong \mathrm{g}_{x, \frac{1}{m}}^{\mathrm{Fr}} \stackrel{\lambda}{\longrightarrow} \mathfrak{f} \stackrel{\psi}{\longrightarrow} \mathrm{C}^{\times} .
$$

The inflation of $\psi_{\lambda}$ to $G_{x, \frac{1}{m}}$ will also be denoted by $\psi_{\lambda}$.

Let

$$
H_{x, \lambda}:=\operatorname{Stab}_{G_{[x]}}(\lambda), \quad \mathrm{S}_{x, \lambda}:=H_{x, \lambda} / G_{x, \frac{1}{m}} .
$$

Then $H_{x, \lambda}$ is the stabilizer of $\psi_{\lambda}$ in $G_{[x]}$.

We will assume that $\lambda$ is $\mathrm{G}_{x}$-stable in $\check{\mathrm{g}}_{x, \frac{1}{m}}$. In all the cases that we will consider in this paper, $\mathbf{S}_{x, \lambda}$ is a finite abelian group. Note that the order of $\mathbf{S}_{x, \lambda}$ is prime to $p$ and $G_{x, \frac{1}{m}}$ is pro- $p$.

Proposition 2.3.1. The group $\mathrm{S}_{x, \lambda}$ splits in $H_{x, \lambda}$, i.e. $H_{x, \lambda}=\mathrm{S}_{x, \lambda} \ltimes G_{x, \frac{1}{m}}$.

Proof. We shall show that $\mathrm{S}_{x, \lambda}$ splits in $H_{x, \lambda} / G_{x, \frac{i}{m}}$ for all $i \geq 1$. For $i=1$ there is nothing to prove. Assume that we have constructed a splitting $s_{i}: \mathrm{S}_{x, \lambda} \rightarrow H_{x, \lambda} / G_{x, \frac{i}{m}}$. The obstruction to lift this splitting to $H_{x, \lambda} / G_{x, \frac{i+1}{m}}$ lies in $H^{2}\left(\mathrm{~S}_{x, \lambda}, G_{x, \frac{i}{m}: \frac{i+1}{m}}\right)$. Since the order of $\mathrm{S}_{x, \lambda}$ is prime to $p$ and $G_{x, \frac{i}{m}: \frac{i+1}{m}}$ is an elementary $p$-group, this cohomology vanishes. Hence $s_{i}$ can be lifted to $s_{i+1}$ and the proposition follows by passing to a limit.

We extend $\psi_{\lambda}$ to a character of $H_{x, \lambda}$ by setting $\psi_{\lambda}$ to be trivial on $\mathrm{S}_{x, \lambda}$. By $H^{1}\left(\mathrm{~S}_{x, \lambda}, G_{x, \frac{1}{m}}\right)=$ 0 , we know that all splittings are conjugate up to $G_{x, \frac{1}{m}}$-conjugation. Hence the extension $\psi_{\lambda}$ is unique and therefore canonical.

Let $\chi$ be a character of $\mathrm{S}_{x, \lambda}$ and let $\pi_{x}(\lambda, \chi):=\operatorname{ind}_{H_{x, \lambda}}^{G} \psi_{\lambda} \otimes \chi$. By [23, Prop. 5.2], $\pi_{x}(\lambda, \chi)$ is an irreducible supercuspidal representation of $G$. We will call $(x, \lambda, \chi)$ an epipelagic data of order $m$ and we call $\pi_{x}(\lambda, \chi)$ an (irreducible) epipelagic supercuspidal representation attached to the data. It contains a minimal $K$-type represented by a coset $\lambda=[\Gamma]=\Gamma+\mathfrak{g}_{x,-\frac{1}{m}}+$ in $\mathfrak{g}_{x,-\frac{1}{m}:-\frac{1}{m}}+$ where $K=G_{x, \frac{1}{m}}$.

Proposition 2.3.2. Suppose $G$ is a group appearing in a type I reductive dual pair.

(i) All unrefined minimal $K$-types of $\pi_{x}(\lambda, \chi)$ are $G$-conjugate to $\Gamma+\mathfrak{g}_{x,-\frac{1}{m}}{ }^{+}$.

(ii) If $\pi_{x}(\lambda, \chi)$ and $\pi_{x}\left(\lambda^{\prime}, \chi^{\prime}\right)$ are isomorphic $G$-modules, then $\lambda$ and $\lambda^{\prime}$ are in the same $G_{x^{-}}$orbit. In addition if $\lambda=\lambda^{\prime}$ then $\chi=\chi^{\prime}$.

Proof. (i) Let $\Gamma+\mathfrak{g}_{x,-\frac{1}{m}}+$ represent the unrefined minimal $K$-type of $\pi$ as above. We will see in Lemma 7.3.1 later that $\Gamma \in \mathfrak{g}_{x,-\frac{1}{m}}$ is a good element and $\mathbf{H}=\mathrm{Z}_{\mathbf{G}}(\Gamma)$ is a torus. Let $H=\mathbf{H}(k)$ and let $\mathfrak{h}=\mathrm{Z}_{\mathfrak{g}}(\Gamma)$ be its Lie algebra. Since $(x, \Gamma, \chi)$ is a tamely ramified supercuspidal data, $H / \mathrm{Z}(G)$ is $k$-anisotropic (or see for example, [12, Proposition 14.5]). We are considering type I classical dual pairs so $\mathrm{Z}(G)$ is anisotropic. Therefore $H$ is $k$-anisotropic. Hence $\mathcal{B}(\mathbf{H}, k)=\{x\}$. 
Suppose $\Gamma_{y}+\mathfrak{g}_{y,-\frac{1}{m}}+$ is another unrefined minimal $K$-type of $\pi$ for some $y \in \mathcal{B}(\mathbf{G}, k)$. Now we show that we can move $y$ to $x$. Since $\Gamma+\mathfrak{g}_{x,-\frac{1}{m}}+$ and $\Gamma_{y}+\mathfrak{g}_{y,-\frac{1}{m}}+$ are minimal $K$-types of $\pi$, they are associates [19], i.e. there exists a $g \in G$ such that

$$
{ }^{g}\left(\Gamma_{y}+\mathfrak{g}_{y,-\frac{1}{m}}{ }^{+}\right) \cap\left(\Gamma+\mathfrak{g}_{x,-\frac{1}{m}}{ }^{+}\right)=\left({ }^{g} \Gamma_{y}+\mathfrak{g}_{g y,-\frac{1}{m}}{ }^{+}\right) \cap\left(\Gamma+\mathfrak{g}_{x,-\frac{1}{m}}{ }^{+}\right)
$$

is nonempty.

Now we apply the argument in the proof of [13, Corollary 2.4.8]. By (2), there are $X^{\prime \prime} \in \mathfrak{g}_{x,-\frac{1}{m}}{ }^{+}$and $Y^{\prime \prime} \in \mathfrak{g}_{g y,-\frac{1}{m}}{ }^{+}$such that ${ }^{g} \Gamma_{y}+Y^{\prime \prime}=\Gamma+X^{\prime \prime}$. By [13, Corollary 2.3.5], there exists $h \in G_{x, 0^{+}}$such that ${ }^{h}\left(\Gamma+X^{\prime \prime}\right)=\Gamma+X^{\prime} \in\left(\Gamma+\mathfrak{g}_{x,-\frac{1}{m}}{ }^{+}\right) \cap \mathfrak{h} \cap \mathfrak{g}_{h g y,-\frac{1}{m}}$ where $X^{\prime} \in \mathfrak{h}_{x,-\frac{1}{m}}{ }^{+}$. By [13, Lemma 2.4.7] $h g y \in \mathcal{B}(\mathbf{H}, k)=\{x\}$, i.e. $h g y=x$. We consider the isomorphism $\operatorname{Ad}\left((h g)^{-1}\right): \mathfrak{g}_{y,-\frac{1}{m}} / \mathfrak{g}_{y,-\frac{1}{m}}{ }^{+} \stackrel{\sim}{\longrightarrow} \mathfrak{g}_{x,-\frac{1}{m}} / \mathfrak{g}_{x,-\frac{1}{m}}{ }^{+}$. It is now clear that the coset $\left.{ }^{h g} \Gamma_{y}\right]=[\Gamma]=\lambda$. This proves $(\mathrm{i})$.

(ii) The last assertion of (ii) is [23, Lemma 2.2]. Now we prove the first assertion. Note that $\lambda=\Gamma+\mathfrak{g}_{x,-\frac{1}{m}}{ }^{+}$and $\lambda^{\prime}=\Gamma^{\prime}+\mathfrak{g}_{x,-\frac{1}{m}}+$ represent unrefined minimal $K$-types of $\pi_{x}(\lambda, \chi)$ and $\pi_{x}\left(\lambda^{\prime}, \chi^{\prime}\right)$ respectively. Since $\pi_{x}(\lambda, \chi) \cong \pi_{x}\left(\lambda^{\prime}, \chi^{\prime}\right)$, the two minimal $K$-types are associates. By the proof in (i) where $y=x$ and $\Gamma_{y}=\Gamma^{\prime}$, we conclude that there exists $g \in G$ such that $g x=x$ and $g \lambda^{\prime}=\lambda$. In particular $g \in G_{x}$. Hence $\lambda$ and $\lambda^{\prime}$ are in the

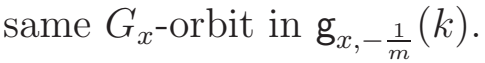

\section{Classical Reductive dual pairs and local theta correspondence}

3.1. Classical groups. In this section, we will define the classical groups which appear in the irreducible dual pairs.

Let $D$ be a division algebra over $k$ with an involution $\tau$ in one of the following cases:

(i) $D=k, \tau$ is the identity map on $k$ and $\varpi_{D}=\varpi$.

(ii) $D$ is a quadratic extension of $k, \tau$ is the nontrivial Galois element in $\operatorname{Gal}(D / k), \varpi_{D}$ is a uniformizer of $D$ so that $\varpi_{D}=\varpi$ if $D / k$ is unramified or $\tau\left(\varpi_{D}\right)=-\varpi_{D}$ if it is ramified.

(iii) $D$ is the quaternion algebra over $k, \tau$ is the usual involution on $D, \varpi_{D}$ is a uniformizer of $D$ such that $\varpi_{D}^{2}=\varpi$.

Let $\mathfrak{o}_{D}$ denote the ring of integers of $D, \mathfrak{p}_{D}=\varpi_{D} \mathfrak{o}_{D}$ denote its maximal prime ideal and $\mathfrak{f}_{D}=\mathfrak{o}_{D} / \mathfrak{p}_{D}$ denote its residue field. We set $\nu_{D}:=\nu\left(\varpi_{D}\right)$.

Let $V$ be a right $D$-vector space. Let $\operatorname{End}_{D}(V)$ denote the space of $D$-linear endomorphisms of $V$ which acts on the left. For $\epsilon= \pm 1$, let $\langle,\rangle_{V}: V \times V \rightarrow D$ be an $\epsilon$-Hermitian sesquilinear form, i.e.

$$
\left\langle v_{1}, v_{2}\right\rangle_{V}=\epsilon\left\langle v_{2}, v_{1}\right\rangle_{V}^{\tau} \quad \text { and } \quad\left\langle v_{1} a_{1}, v_{2} a_{2}\right\rangle_{V}=a_{1}^{\tau}\left\langle v_{1}, v_{2}\right\rangle_{V} a_{2}
$$

for all $v_{1}, v_{2} \in V$ and $a_{1}, a_{2} \in D$. The $\epsilon$-Hermitian form induces a conjugation $*: \operatorname{End}_{D}(V) \rightarrow$ $\operatorname{End}_{D}(V)$ such that $\left\langle g v_{1}, v_{2}\right\rangle_{V}=\left\langle v_{1}, g^{*} v_{2}\right\rangle_{V}$ for all $v_{1}, v_{2} \in V$ and $g \in \operatorname{End}_{D}(V)$. Then

$$
\begin{aligned}
& G=\mathrm{U}(V)=\mathrm{U}\left(V,\langle,\rangle_{V}\right):=\left\{g \in \operatorname{End}_{D}(V) \mid g g^{*}=\mathrm{Id}\right\} \quad \text { and } \\
& \mathfrak{g}=\mathfrak{u}(V)=\mathfrak{u}\left(V,\langle,\rangle_{V}\right):=\left\{X \in \operatorname{End}_{D}(V) \mid X+X^{*}=0\right\}
\end{aligned}
$$

are a classical group and its Lie algebra.

3.2. Irreducible reductive dual pairs of type I. Let $V$ be a right $D$-vector space equipped with an $\epsilon$-Hermitian sesquilinear form $\langle,\rangle_{V}$ and let $V^{\prime}$ be a right $D$-vector space equipped with an $\epsilon^{\prime}$-Hermitian sesquilinear form $\langle,\rangle_{V^{\prime}}$ where $\epsilon^{\prime}=-\epsilon$. Let $G$ and $G^{\prime}$ be the classical groups defined by $\left(V,\langle,\rangle_{V}\right)$ and $\left(V^{\prime},\langle,\rangle_{V^{\prime}}\right)$ respectively. 
We view $V^{\prime}$ as a left $D$-module by $a v=v a^{\tau}$ for all $a \in D$ and $v \in V^{\prime}$. Let $W=V \otimes_{D} V^{\prime}$. It is a symplectic $k$-vector space with symplectic form $\langle$,$\rangle given by$

$$
\left\langle v_{1} \otimes v_{1}^{\prime}, v_{2} \otimes v_{2}^{\prime}\right\rangle=\operatorname{tr}_{D / k}\left(\left\langle v_{1}, v_{2}\right\rangle_{V}\left\langle v_{1}^{\prime}, v_{2}^{\prime}\right\rangle_{V^{\prime}}^{\tau}\right) \text {. }
$$

Then $G$ and $G^{\prime}$ commute with each other in the symplectic group $\operatorname{Sp}(W)$. We call $\left(G, G^{\prime}\right)$ an irreducible reductive dual pair of type $I$.

3.3. Lattice model. We recall that $\psi: k \rightarrow \mathrm{C}^{\times}$is a non-trivial additive character with conductor $\mathfrak{p}_{k}$. Let $A$ be a self dual lattice in $W$, i.e. $A=\left\{w \in W \mid\left\langle w, w^{\prime}\right\rangle \in \mathfrak{p}_{k}, \forall w^{\prime} \in A\right\}$. The lattice model with respect to $A$ of the oscillator representation $\omega$ with respect to the character $\psi$ is defined by

$$
\mathscr{S}(A)=\left\{\begin{array}{l|c}
f: W \rightarrow \mathrm{C} & \begin{array}{c}
f(a+w)=\psi\left(\frac{1}{2}\langle w, a\rangle\right) f(w) \forall a \in A \\
f \text { locally constant, compactly supported }
\end{array}
\end{array}\right\} .
$$

Let $\operatorname{Mp}(W)$ be the metaplectic $\mathrm{C}^{\times}$-covering of $\operatorname{Sp}(W)$ which acts on the oscillator representation naturally by its definition. The lattice model with respect to $A$ gives a section $\omega_{A}: \operatorname{Sp}(W) \hookrightarrow \operatorname{Mp}(W)$ of the natural projection $\operatorname{Mp}(W) \rightarrow \operatorname{Sp}(W)$ (c.f. [17, 27]). Let $\operatorname{Sp}_{A}:=\operatorname{Stab}_{\operatorname{Sp}(W)}(A)=\{g \in \operatorname{Sp}(W) \mid g A \subseteq A\}$. We only describe $\omega_{A}(g)$ for $g \in \operatorname{Sp}_{A}$ :

$$
\left(\omega_{A}(g) f\right)(w)=f\left(g^{-1} w\right) \quad \forall g \in \operatorname{Sp}_{A}, f \in \mathscr{S}(A) \text { and } w \in W .
$$

The splitting $\omega_{A}$ does not depend on the choice of the self-dual lattice $A$. More precisely, we have the following proposition which follows immediately from Lemma C.1.1.

Proposition 3.3.1. There is a section

$$
\omega_{0}: \bigcup_{A \text { is self-dual }} \operatorname{Sp}_{A} \longrightarrow \operatorname{Mp}(W)
$$

such that $\left.\omega_{0}\right|_{\operatorname{Sp}_{A}}=\omega_{A}$ for every self dual lattice $A$.

3.4. Epipelagic supercuspidal representations of covering groups. Let $\Sigma=(x, \lambda, \chi)$ be an epipelagic datum of order $m$. We retain the notation for the subgroup $H_{x, \lambda}=$ $\mathrm{S}_{x, \lambda} \ltimes G_{x, \frac{1}{m}}$ and its character $\psi_{\lambda} \otimes \chi$ in Section 2.3. Since $G$ is a member of a type I dual pair, we recall that $G_{x}=G_{[x]}$ in Section 1.3 and $H_{x, \lambda}$ is a subgroup of $G_{x}$. We will show in Section 8.2 later that $G_{x}$ stabilizes a self-dual lattice $A$ in $W$. Then Proposition 3.3.1 gives a splitting

$$
\left.\omega_{0}\right|_{G_{x}}: G_{x} \longrightarrow \widetilde{G}_{x}
$$

of $\widetilde{G}_{x} \rightarrow G_{x}$. We will identify $H_{x, \lambda}$ and $G_{x}$ as subgroups of $\widetilde{G}_{x}$ via $\omega_{0}$. Let $\operatorname{id}_{\mathrm{C} \times}: \mathrm{C}^{\times} \rightarrow \mathrm{C}^{\times}$ be the identity map. Under this splitting, $\widetilde{G}_{x}=G_{x} \times \mathrm{C}^{\times}$with $\mathrm{C}^{\times}$acting on the oscillator representation $\mathscr{S}$ via $\operatorname{id}_{\mathrm{C}} \times$. Now

$$
\pi_{\Sigma}:=\operatorname{ind}_{H_{x, \lambda} \times \mathrm{C}^{\times}}^{\widetilde{G}}\left(\left(\psi_{\lambda} \otimes \chi\right) \otimes \operatorname{id}_{\mathrm{C} \times}\right)
$$

is an irreducible supercuspidal representation of $\widetilde{G}$ which is also denoted by $\pi_{\Sigma}^{\widetilde{G}}$ or $\pi_{x}^{\widetilde{G}}(\lambda, \chi)$. We will also call $\pi_{\Sigma}$ an epipelagic supercuspidal representation attached to the epipelagic data $\Sigma$.

By Appendix C.2 the splitting of $G_{x, 0^{+}}$is canonically defined for any $x \in \mathcal{B}(\mathbf{G}, k)$. In particular, it still makes sense to talk about positive depth minimal $K$-types. In addition Proposition 2.3.2 holds if we replace $\pi_{x}(\lambda, \chi)$ with $\pi_{\Sigma}^{\widetilde{G}}$ without any modification.

\section{Bruhat-Tits Buildings and Moy-Prasad filtrations of Classical groups}

In this section we recall some known facts about the Bruhat-Tits buildings of classical groups. Our references are [3, 5, 14]. 
4.1. Lattice functions. A (right) $\mathfrak{o}_{D}$-lattice $L$ in a right $D$-vector space $V$ is a right $\mathfrak{o}_{D}$-submodule such that $L \otimes_{\mathfrak{o}_{D}} D=V$.

Definition 4.1.1. $\quad 1$. Let Latt $_{V}$ be the set of $\mathfrak{o}_{D}$-lattice valued functions $s \mapsto \mathscr{L}_{s}$ on $\mathrm{R}$ such that (i) $\mathscr{L}_{s} \supseteq \mathscr{L}_{t}$ if $s<t$, (ii) $\mathscr{L}_{s+\nu_{D}}=\mathscr{L}_{s} \varpi_{D}$ and (iii) $\mathscr{L}_{s}=\bigcap_{t<s} \mathscr{L}_{t}$.

2. We set $\mathscr{L}_{r^{+}}:=\bigcup_{t>r} \mathscr{L}_{t}$ and $\operatorname{Jump}(\mathscr{L})=\left\{r \in \mathrm{R} \mid \mathscr{L}_{r} \supsetneq \mathscr{L}_{r^{+}}\right\}$.

3. Given any lattice function $\mathscr{L}$, we define

$$
\begin{aligned}
\mathfrak{g l}(V)_{\mathscr{L}, r} & :=\left\{X \in \mathfrak{g l}(V) \mid X \mathscr{L}_{s} \subseteq \mathscr{L}_{s+r}, \forall s \in \mathrm{R}\right\} & & \forall r \in \mathrm{R}, \\
\operatorname{GL}(V)_{\mathscr{L}, r} & :=\left\{g \in \mathrm{GL}(V) \mid(g-1) \mathscr{L}_{s} \subseteq \mathscr{L}_{s+r}, \forall s \in \mathrm{R}\right\} & & \forall r>0, \\
\mathrm{GL}(V)_{\mathscr{L}} & :=\left\{g \in \mathrm{GL}(V) \mid g \mathscr{L}_{s} \subseteq \mathscr{L}_{s}\right\} . & &
\end{aligned}
$$

4. For $r<s$, we denote $\mathscr{L}_{r: s}=\mathscr{L}_{r} / \mathscr{L}_{s}$.

Definition 4.1.2. $\quad$ 1. A $D$-norm of $V$ is a function $l: V \rightarrow \mathrm{R} \cup\{\infty\}$ such that for all $x, y \in V$ and $d \in D$, (i) $l(x d)=l(x)+\nu(d)$, (ii) $l(x+y) \geq \min (l(x), l(y))$ and (iii) $l(x)=\infty$ if and only if $x=0$.

2. The norm $l$ is called splittable if there is a $D$-basis $\left\{e_{i} \mid i \in I\right\}$ of $V$ such that $l\left(\sum_{i \in I} e_{i} d_{i}\right)=\inf _{i \in I}\left(l\left(e_{i}\right)+\nu\left(d_{i}\right)\right)$. Let $\mathcal{S N}(V)$ denote the splittable norms on $V$. In this paper, all norms refer to splittable $D$-norms.

There is a natural bijection between $\mathcal{S N}(V)$ and $\operatorname{Latt}_{V}$ given by $l \mapsto\left(\mathscr{L}_{r}=l^{-1}([r,+\infty))\right)$. Then $\operatorname{Jump}(\mathscr{L})$ is the image of $l$.

The following theorem is well known and follows directly from the definition of MoyPrasad filtration [19].

Theorem 4.1.3. The (extended) building $\mathcal{B}(\mathrm{GL}(V))$ could be identified with $\mathrm{Latt}_{V}$ as $\mathrm{GL}(V)$-sets. This identification is unique up to translation (c.f. [4, Theorem 2.11]). Suppose $x \in \mathcal{B}(\mathrm{GL}(V))$ corresponds to the lattice function $\mathscr{L} \in \mathrm{Latt}_{V}$. Then

(a) $\mathfrak{g l}(V)_{x, r}=\mathfrak{g l}(V)_{\mathscr{L}, r}$ for $r \in \mathrm{R}$,

(b) $\mathrm{GL}(V)_{x, r}=\mathrm{GL}(V)_{\mathscr{L}, r}$ for $r>0$ and

(c) $\mathrm{GL}(V)_{\mathscr{L}}=\mathrm{GL}(V)_{x}$.

For the rest of this paper, we will freely interchange the notion of points in the building of $\mathrm{GL}(V), D$-norms and lattice functions.

4.2. Tensor products. Suppose $l$ and $l^{\prime}$ are two norms on $D$-modules $V$ and $V^{\prime}$. Then there is an induced norm on $W:=V \otimes_{D} V^{\prime}$ such that $\left(l \otimes l^{\prime}\right)\left(v \otimes v^{\prime}\right)=l(v)+l^{\prime}\left(v^{\prime}\right)$ (c.f. [4, $\S 1.11]$ ). Let $\mathscr{L}$ and $\mathscr{L}^{\prime}$ be the corresponding lattice functions. We denote by $\mathscr{L} \otimes \mathscr{L}^{\prime}$ the corresponding $\mathfrak{o}_{k}$-lattice function on $V \otimes_{D} V^{\prime}$ where

$$
\left(\mathscr{L} \otimes \mathscr{L}^{\prime}\right)_{t}=\sum_{r+r^{\prime}=t} \mathscr{L}_{r} \otimes_{\mathfrak{o}_{D}} \mathscr{L}_{r^{\prime}}^{\prime}
$$

It is easy to see that

$$
\operatorname{Jump}\left(\mathscr{L} \otimes \mathscr{L}^{\prime}\right)=\operatorname{Jump}(\mathscr{L})+\operatorname{Jump}\left(\mathscr{L}^{\prime}\right)
$$

The norms $l$ and $l^{\prime}$ also induce a natural norm $\operatorname{Hom}\left(l, l^{\prime}\right)$ on $\operatorname{Hom}_{D}\left(V, V^{\prime}\right)$ whose corresponding lattice function is

$$
\left(\operatorname{Hom}\left(\mathscr{L}, \mathscr{L}^{\prime}\right)\right)_{r}:=\left\{w \in \operatorname{Hom}_{D}\left(V, V^{\prime}\right) \mid w\left(\mathscr{L}_{s}\right) \subseteq \mathscr{L}_{s+r}^{\prime} \forall s \in \mathrm{R}\right\} .
$$

In particular, every norm $l$ on $V$ defines a dual norm $l^{*}:=\operatorname{Hom}(l, \nu)$ on $V^{*}:=\operatorname{Hom}_{D}(V, D)$. Under the isomorphism $\operatorname{Hom}_{D}\left(V, V^{\prime}\right) \cong V^{\prime} \otimes_{D} V^{*}$, the norms $\operatorname{Hom}\left(l, l^{\prime}\right)$ and $l^{\prime} \otimes l^{*}$ coincide. If $V=V^{\prime}$, then the Moy-Prasad lattice function $r \mapsto \mathfrak{g l}(V)_{r}$ defined in Definition 4.1.1 is the tensor product lattice function $\mathscr{L} \otimes \mathscr{L}^{*}$ on $\mathfrak{g l}(V)=\operatorname{End}_{D}(V)$. 
4.3. Self-dual lattice functions. Let $V$ be a space with a non-degenerate sesquilinear form $\langle,\rangle_{V}$.

Definition 4.3.1. $\quad$ 1. For a lattice $L$ in $\mathrm{V}$, we set

$$
L^{\sharp}:=\left\{v \in V \mid\left\langle v, v^{\prime}\right\rangle_{V} \in \mathfrak{p}_{D}, \forall v^{\prime} \in L\right\} .
$$

A lattice $L$ is called self-dual if $L=L^{\sharp}$. A lattice $L$ is called good if $L^{\sharp} \mathfrak{p}_{D} \subseteq L \subseteq L^{\sharp}$.

2. For a lattice function $\mathscr{L}$ we define its dual lattice function $\mathscr{L}^{\sharp}$ by $\left(\mathscr{L}^{\sharp}\right)_{s}=\left(\mathscr{L}_{(-s)^{+}}\right)^{\sharp}$. If $l$ is the norm corresponding to $\mathscr{L}$, then we denote the norm corresponding to $\mathscr{L}^{\sharp}$ by $l^{\sharp}$. If we identify $V$ with $V^{*}$ using the form $\langle,\rangle_{V}$, then the norm $l^{*}$ on $V^{*}$ translates to the norm $l \sharp$ on $V$.

3. A lattice function $\mathscr{L}$ is called self-dual if and only if $\mathscr{L}=\mathscr{L}^{\sharp}$. In terms of norm, it is equivalent to $l^{\sharp}=l$ (c.f. [3, Prop. 3.3]) and we say that $l$ is self-dual. Let Latt $t_{V}^{\sharp}$ be the set of self-dual lattice functions. Clearly Latt $\|_{V}^{\sharp}$ is the $\sharp$-fixed point set of $\operatorname{Latt}_{V}$.

4. When $\mathscr{L}$ is self-dual, we define $\mathfrak{g}_{\mathscr{L}, r}:=\mathfrak{g} \cap \mathfrak{g l}(V)_{\mathscr{L}, r}, G_{\mathscr{L}, r}:=G \cap \operatorname{GL}(V)_{\mathscr{L}, r}$, $G_{\mathscr{L}}:=G \cap \mathrm{GL}(V)_{\mathscr{L}}, \mathrm{G}_{\mathscr{L}}:=G_{\mathscr{L}} / G_{\mathscr{L}, 0^{+}}$and $\mathfrak{g}_{\mathscr{L}, r: s}:=\mathfrak{g}_{\mathscr{L}, r} / \mathfrak{g}_{\mathscr{L}, s}$

Remark. If we identify $V^{*} \otimes_{D} V^{*}$ as $\left(V \otimes_{D} V^{\prime}\right)^{*}$, then by a calculation on a splitting basis, we have $\left(l \otimes l^{\prime}\right)^{*}=l^{*} \otimes l^{\prime *}$ (c.f. [4, (18),(21), Sect. 1.12]). In particular, suppose that $V$ and $V^{\prime}$ are formed spaces, and $\mathscr{L}$ and $\mathscr{L}^{\prime}$ are self-dual lattice functions. It is easy to see that $\left(l_{\mathscr{L}} \otimes l_{\mathscr{L}^{\prime}}\right)^{\sharp}=l_{\mathscr{L}} \otimes l_{\mathscr{L}^{\prime}}$, i.e. it is self-dual. Hence $\mathscr{L} \otimes \mathscr{L}^{\prime}$ is a self-dual lattice on $V \otimes_{D} V^{\prime}$.

4.4. We recall that $k$ is a $p$-adic field with $p \neq 2$. For a classical group $G$ defined over $k$, $\mathcal{B}(\mathbf{G}, k)$ could be identified canonically with the set of splittable self-dual norms on $V$ (c.f. [5, 8]). The following theorem is the culmination of [5], [3], [14] and [8].

Theorem 4.4.1. (i) There is a natural $G$-equivariant bijection between $\mathcal{B}(\mathbf{G}, k)$ and Latt $_{V}^{\sharp}$.

(ii) Suppose $x \in \mathcal{B}(\mathbf{G}, k)$ corresponds to $\mathscr{L} \in$ Latt $_{V}^{\sharp}$. Then

(a) $\mathfrak{g}_{\mathscr{L}, r}=\mathfrak{g}_{x, r}$ for $r \in \mathrm{R}$,

(b) $G_{\mathscr{L}, r}=G_{x, r}$ for $r>0$ and

(c) $G_{\mathscr{L}}=G_{x}$.

4.5. Let $r \in \operatorname{Jump}(\mathscr{L})$ so that $\mathrm{L}_{r}:=\mathscr{L}_{r: r^{+}}=\mathscr{L}_{r} / \mathscr{L}_{r^{+}}$which is nonzero. The sesquilinear form $\langle,\rangle_{V}$ induces a nonzero pairing $\mathscr{L}_{r} \times \mathscr{L}_{-r} \rightarrow \mathfrak{o}_{D}$ and a non-degenerate pairing over $\mathfrak{f}_{D}$ :

$$
\mathrm{L}_{r} \times \mathrm{L}_{-r} \rightarrow \mathfrak{f}_{D}
$$

In particular we have

$$
\operatorname{Jump}(\mathscr{L})=-\operatorname{Jump}(\mathscr{L})
$$

The structure of $\mathrm{G}_{\mathscr{L}}$ is described in the following lemma. It is well known so we omit its proof. Also see Appendix A

Lemma 4.5.1. Let $\nu_{D}=\nu\left(\varpi_{D}\right)$. Then

$$
\mathrm{G}_{\mathscr{L}} \cong \mathrm{G}_{0} \times \mathrm{G}_{\frac{1}{2} \nu_{D}} \times \prod_{r \in \operatorname{Jump}(\mathscr{L}) \cap\left(0, \frac{1}{2} \nu_{D}\right)} \mathrm{GL}\left(\mathrm{L}_{r}\right)
$$

where $\mathrm{G}_{0} \cong \mathrm{U}\left(\mathrm{L}_{0}\right)$ and $\mathrm{G}_{\frac{1}{2} \nu_{D}} \cong \mathrm{U}\left(\mathrm{L}_{\frac{1}{2} \nu_{D}}\right)$ where $\mathrm{L}_{\frac{1}{2} \nu_{D}}$ is equipped with the form $\left\langle\left[v_{1}\right],\left[v_{2}\right]\right\rangle=$ $\left\langle v_{1}, v_{2} \varpi_{D}^{-1}\right\rangle_{V}\left(\bmod \mathfrak{p}_{D}\right)$. 


\section{TAME BASE Changes AND EPIPElagic POINTS}

5.1. Let $D$ be the division algebra over $k$ as in Section 3.1. Let $V$ be a $D$-module with an $\epsilon$-Hermitian sesquilinear form $\langle,\rangle_{V}$ and let $\mathbf{G}$ be the classical group defined over $k$ such that $G:=\mathbf{G}(k)=\mathrm{U}\left(V,\langle,\rangle_{V}\right)$. Suppose $E$ is a tamely ramified finite extension of $k$ or $k^{\text {ur }}$ such that $\mathbf{G}$ splits. In this section we study the relations between buildings under tamely ramified field extensions.

In all cases, it is standard to construct an $E$-vector space $\breve{V}$ obtained by certain base change of $V$ so that $\mathbf{G}(k) \subseteq \mathbf{G}(E)$ are subgroups of $\mathrm{GL}_{E}(\breve{V})$. By [8] there is a canonical bijection $\mathcal{B}(\mathbf{G}, k) \sim \mathcal{B}(\mathbf{G}, E)^{\operatorname{Gal}(E / k)}$. The next proposition describes this bijection in terms of splittable norms on $V$ and $\breve{V}$.

Proposition 5.1.1. We identify buildings of classical groups with the corresponding set of splittable norms.

(i) Suppose $D=k$. Let $\breve{V}=V \otimes_{k} E$ and let $i_{V}: V \rightarrow \breve{V}$ be given by $v \mapsto v \otimes 1$. Let $\langle,\rangle_{\breve{V}}$ be the E-linear extension of $\langle,\rangle_{V}$. Then $\mathbf{G}(E)=\mathrm{U}\left(\breve{V},\langle,\rangle_{\breve{V}}\right)$. The bijection $\mathcal{B}(\mathbf{G}, k) \stackrel{\sim}{\sim}(\mathbf{G}, E)^{\operatorname{Gal}(E / k)}$ is given by $l_{V} \mapsto l_{V} \otimes_{k}\left(\left.\nu\right|_{E}\right)$ and its inverse map is $l_{\breve{V}} \mapsto$ $l_{\breve{V}} \circ i_{V}$

(ii) Suppose $D$ is a quadratic extension of $k$. We fix a field embedding $\iota \in \operatorname{Hom}_{k}(D, E)$ and view $D$ as a subfield of $E$. Let $\breve{V}=V \otimes_{D} E$ and let $i_{V}: V \rightarrow \breve{V}$ be given by $v \mapsto v \otimes 1$. Then $\mathbf{G}(E) \cong \mathrm{GL}_{E}(\breve{V})$. The bijection $\mathcal{B}(\mathbf{G}, k) \stackrel{\sim}{\sim}(\mathbf{G}, E)^{\operatorname{Gal}(E / k)}$ is given by $l_{V} \mapsto l_{V} \otimes_{D}\left(\left.\nu\right|_{E}\right)$ and its inverse map is $l_{\breve{V}} \mapsto l_{\breve{V}} \circ i_{V}$.

(iii) Suppose $D$ is the quaternion algebra over $k$. We fix a subfield $L$ of $E$ which is a quadratic extension of $k$. We identify $L$ with a subfield of $D$ and fix a $d \in D$ such that $d^{2} \in k^{\times}, d^{\tau}=-d$ and $\operatorname{Ad}(d)$ acts on $L$ by the non-trivial Galois action. Let $\mathrm{pr}: D \rightarrow L$ be the projection of $D=L \oplus L d$. Then $Q\left(v_{1}, v_{2}\right):=\operatorname{pr}\left(\left\langle v_{1} d, v_{2}\right\rangle_{V}\right)$ defines an L-bilinear from on $V$. Let $\breve{V}=V \otimes_{L} E$ and let $\langle,\rangle_{\breve{V}}$ be the $(-\epsilon)$-symmetric E-linear extension of $Q$. Then $\mathbf{G}(E) \cong \mathrm{U}\left(\breve{V},\langle,\rangle_{\breve{V}}\right)$. The bijection $\mathcal{B}(\mathbf{G}, k) \stackrel{\sim}{\longrightarrow} \mathcal{B}(\mathbf{G}, E)^{\operatorname{Gal}(E / k)}$ is given by $l_{V} \mapsto l_{V} \otimes_{L}\left(\left.\nu\right|_{E}\right)$ and its inverse map is $l_{\breve{V}} \mapsto l_{\breve{V}} \circ i_{V}$.

Before we give the proof of Proposition 5.1.1, we first recall the uniqueness result stated in $[8, \S 1.2]$.

Lemma 5.1.2. Let $\mathcal{B}$ and $\mathcal{B}^{\prime}$ be two $G$-sets satisfying the axioms of building of $\mathbf{G}$ over $k$ (See [25, §2.1] and [8, §1.9.1].). Let $j: \mathcal{B} \rightarrow \mathcal{B}^{\prime}$ be a bijection such that

(i) $j$ is G-equivariant, i.e. $j(g \cdot x)=g \cdot j(x)$ for all $g \in G$, and

(ii) its restriction to an apartment $\mathcal{A}$ is affine.

Then $j$ is unique up to the translation by an element in $X_{*}\left(\mathrm{Z}(G)^{\circ}\right) \otimes \mathrm{R}$.

In our cases, $\mathrm{Z}(G)$ is anisotropic so the map $j$ is unique.

Proof of Proposition 5.1.1. (i) We consider the following diagram:

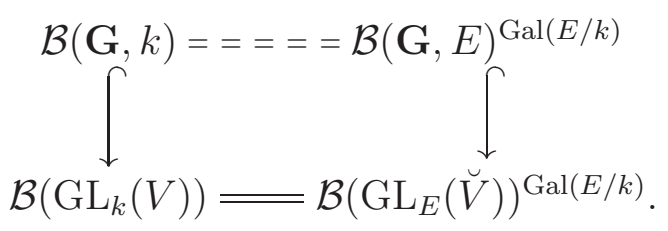

The buildings in the top row are the fixed point sets of the involutions $\sharp$ of the buildings in the bottom row. The bottom map is $l_{V} \mapsto l_{V} \otimes\left(\left.\nu\right|_{E}\right)$. It is a $\mathrm{GL}_{k}(V)$-invariant map. It is a bijection, since it suffices to check this on an apartment, where it is obvious. The map sends self-dual norms to self-dual norms, hence it induces the top row isomorphism, by 
restriction. It is the canonical isomorphism $\mathcal{B}(\mathbf{G}, k) \stackrel{\sim}{\longrightarrow}(\mathbf{G}, E)^{\operatorname{Gal}(E / k)}$ by Lemma 5.1.2. This proves (i).

(ii) We refer to the computation in [4, $\S 1.13]$. Let $\iota_{1}, \iota_{2}$ be two $k$-embeddings of $D$ into $E$ (so $\iota$ is one of the two). Let $\breve{V}^{\iota_{i}}=V \otimes_{D, \iota_{i}} E$. Then $V \otimes_{k} E \cong \breve{V}^{\iota_{1}} \oplus \breve{V}^{\iota_{2}}$, so we have a natural action of $\operatorname{Gal}(E / k)$ on $\breve{V}^{\iota_{1}} \oplus \breve{V}^{\iota_{2}}$. Now Part (ii) follows by applying a similar argument as in (i) to the following diagram:

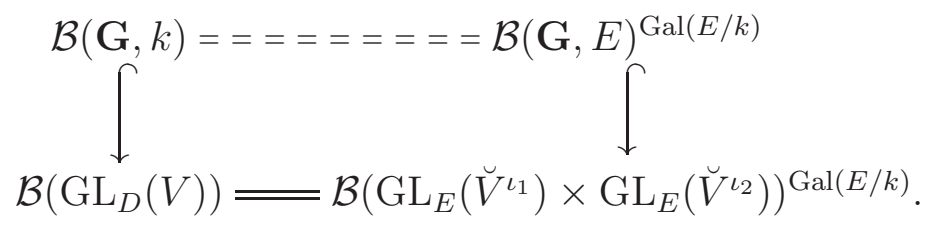

(iii) Note that $H\left(v_{1}, v_{2}\right)=\operatorname{pr}\left(\left\langle v_{1}, v_{2}\right\rangle_{V}\right)$ defines a Hermitian form on $V$. Moreover, $\mathrm{G}(k)=\mathrm{U}(V, Q) \cap \mathrm{U}(V, H)$. Part (iii) follows by applying a similar argument as in (i) to the following diagram:

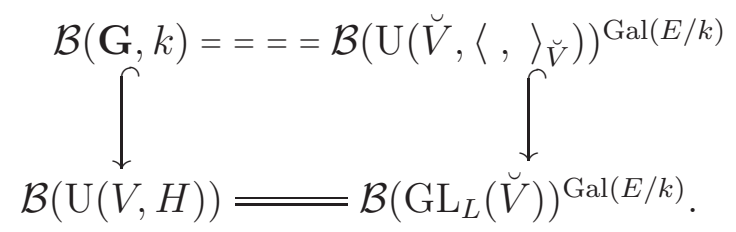

For an $\mathfrak{o}_{D}$-lattice function $\mathscr{L}$ corresponding to $x \in \mathcal{B}(\mathbf{G}, k)$, we will denote by $\mathscr{L}^{E}$ the $\mathfrak{o}_{E}$-lattice function in $\breve{V}$ corresponding to $x \in \mathcal{B}(\mathbf{G}, E)^{\mathrm{Gal}(E / k)}$ in the above proposition. We need the following application of Lemma 5.1.2 in our study of the epipelagic points.

Lemma 5.1.3. Let $\mathscr{L}$ be the self-dual lattice function corresponding to a point $x$ in $\mathcal{B}(\mathbf{G}, k)$ of order $m$. Suppose $\mathbf{G}$ splits under a tamely ramified extension $E$ with ramification index $m$. Then $\operatorname{Jump}(\mathscr{L})$ is contained in either $\frac{1}{m} \mathrm{z}$ or $\frac{1}{2 m}+\frac{1}{m} \mathrm{Z}$.

Proof. We have $\nu(E)=\frac{1}{m}$ z. Let $\mathscr{L}^{E}$ be the $\mathfrak{o}_{E}$-lattice function. Let $J=\operatorname{Jump}(\mathscr{L})$ and $J^{E}=\operatorname{Jump}\left(\mathscr{L}^{E}\right)$. By [23, $\left.\S 4.2\right], \mathscr{L}^{E}$ corresponds to a hyperspecial point in $\mathbf{G}(E)$. Hence $J^{E}=j_{0}+\frac{1}{m} Z$ for some $j_{0} \in\left[0, \frac{1}{m}\right)$.

By Proposition 5.1.1, we have $J \subseteq J^{E}=J+\frac{1}{m}$ z. Since $\mathscr{L}$ is self-dual, $J=-J$. Hence $-j_{0}+\frac{1}{m} \mathrm{Z}=-J^{E} \subseteq-J+\frac{1}{m} \mathrm{Z} \subseteq J+\frac{1}{m} \mathrm{Z} \subseteq J^{E}+\frac{1}{m} \mathrm{Z}=j_{0}+\frac{1}{m} \mathrm{Z}$. Therefore $j_{0}=0$ or $\frac{1}{2 m}$. The lemma follows.

5.2. Epipelagic points. Let $x \in \mathcal{B}(\mathbf{G}, k)$ be an epipelagic point of order $m$. We recall that $k^{\text {ur }}$ is the maximal unramified extension of $k$. Let $E$ be the totally ramified extension of $k^{\text {ur }}$ of degree $m$. We fix a uniformizer $\varpi_{E}$ such that $\varpi_{E}^{m}=\varpi$. Let $\operatorname{Gal}\left(E / k^{\mathrm{ur}}\right)=\langle\sigma\rangle$ where $\sigma\left(\varpi_{E}\right)=\zeta \varpi_{E}$ and $\zeta$ is a primitive $m$-th root of unity. Let $\operatorname{Fr} \in \operatorname{Gal}(E / k)$ denote the lift of the Frobenius automorphism in $\operatorname{Gal}\left(k^{\text {ur }} / k\right)$ such that $\operatorname{Fr}\left(\varpi_{E}\right)=\varpi_{E}$. Now $\operatorname{Gal}(E / k)=\langle\mathrm{Fr}, \sigma\rangle$.

The group $\mathbf{G}$ splits over $E[23, \S 4.1]$. We recall Section 1.1 that $\mathbf{T}$ is a maximally $k^{\mathrm{ur}}$-split torus in $\mathbf{G}$ containing $\mathbf{S}$ and defined over $k$. Let $\mathbf{Y}=\mathrm{Z}_{\mathbf{G}}(\mathbf{T})$ be a Cartan subgroup of $\mathbf{G}$. Then $x$ is a hyperspecial point in $\mathcal{A}(\mathbf{Y}, E)$. We have $\mathbf{G}(E)_{x}^{\sigma}=\mathbf{G}\left(k^{\mathrm{ur}}\right)_{x}$, $\mathbf{G}(E)_{x, r}^{\sigma}=\mathbf{G}\left(k^{\mathrm{ur}}\right)_{x, r}$ for $r>0$, and $\varpi_{E}^{d} \mathfrak{g}(E)_{x, 0}=\mathfrak{g}(E)_{x, \frac{d}{m}}$. The building $\mathcal{B}\left(\mathbf{G}, k^{\mathrm{ur}}\right)$ embeds into $\mathcal{B}(\mathbf{G}, E)$ as the $\sigma$-invariant set and

$$
\mathcal{B}(\mathbf{G}, k)=\mathcal{B}\left(\mathbf{G}, k^{\mathrm{ur}}\right)^{\mathrm{Fr}}=\left(\mathcal{B}(\mathbf{G}, E)^{\sigma}\right)^{\mathrm{Fr}} .
$$

We set $D(E)=D \otimes_{k} E$. We equip $D(E)$ with the tensor product norm of valuations of $D$ and $E$. Let $\mathscr{E}$ and $\mathscr{D}$ be the lattice functions in $E$ and $D$ respectively defined 
by their valuations. Then $\mathscr{D} \otimes \mathscr{E}$ is the corresponding lattice function on $D(E)$. Let $\mathfrak{o}_{D(E)}=(\mathscr{D} \otimes \mathscr{E})_{0}, \mathfrak{p}_{D(E)}=(\mathscr{D} \otimes \mathscr{E})_{0^{+}}$and $\mathfrak{f}_{D(E)}=\mathfrak{o}_{D(E)} / \mathfrak{p}_{D(E)}$ which is a semisimple algebra over $\mathfrak{f}_{E}$.

Let $\mathscr{L}$ be the self-dual lattice function in $V$ corresponding to $x \in \mathcal{B}(\mathbf{G}, k)$. Let $\mathscr{L}_{E}:=$ $\mathscr{L} \otimes \mathscr{E}$ be the self-dual $\mathfrak{o}_{k}$-lattice function in $V(E):=V \otimes_{k} E$. In fact it is an $\mathfrak{o}_{D(E)}$-lattice function. We have following situations.

(i) If $D=k$, then $\mathscr{L}_{E}=\mathscr{L}^{E}$.

(ii) If $D$ is a quadratic extension of $k$, then $D(E) \cong E \times E, V(E)=\breve{V}^{\iota_{1}} \oplus \breve{V}^{\iota_{2}}, \mathscr{L}_{E}=$ $\mathscr{L}^{\iota_{1}, E} \oplus \mathscr{L}^{\iota_{2}, E}$ where $\breve{V}^{\iota_{1}}$ and $\breve{V}^{\iota_{2}}, \mathscr{L}^{\iota_{1}, E}$ and $\mathscr{L}^{\iota_{2}, E}$ correspond to the two different $k$-embeddings of $D$ into $E$.

(iii) Suppose $D$ is the quaternion algebra over $k$. We fix a quadratic extension $L$ of $k$ in $E$. Then $D(E) \cong \operatorname{Mat}_{2}(E), V(E)=\breve{V}^{\iota_{1}} \oplus \breve{V}^{\iota_{2}}$ and $\mathscr{L}_{E}=\mathscr{L}^{\iota_{1}, E} \oplus \mathscr{L}^{\iota_{2}, E}$ where $\mathscr{L}^{\iota_{1}, E}$ and $\mathscr{L}^{\iota_{2}, E}$ are $\mathfrak{o}_{E}$-lattice functions corresponding to the two different $k$-embeddings of $L$ into $E$.

Clearly, $\operatorname{Jump}\left(\mathscr{L}_{E}\right)=\operatorname{Jump}\left(\mathscr{L}^{\iota_{i}, E}\right)=\operatorname{Jump}(\mathscr{L})+\frac{1}{m}$ z. The Galois group $\operatorname{Gal}(E / k)$ acts on $V(E)$ by $s(v \otimes x)=v \otimes s(x)$ for $s \in \operatorname{Gal}(E / k), v \in V$ and $x \in E$. For $g \in \mathbf{G}(E)$ and $s \in \operatorname{Gal}(E / k)$, we have $s(g)=s \circ g \circ s^{-1}$ as $D(E)$-linear automorphism on $V(E)$. In Cases (ii) and (iii), under the decomposition, $\mathbf{G}(E)$ acts diagonally on $V(E)=\breve{V}^{\iota_{1}} \oplus \breve{V}^{\iota_{2}}$.

Extending the notation in Definition 4.1.1, we have $\mathbf{G}(E)_{x}=\mathbf{G}(E)_{\mathscr{L}_{E}}, \mathbf{G}(E)_{x, r}=$ $\mathbf{G}(E)_{\mathscr{L}_{E}, r}$ and $\mathfrak{g}(E)_{x, r}=\mathfrak{g}(E)_{\mathscr{L}_{E}, r}$ by Proposition 5.1.1.

5.3. Kac-Vinberg gradings. In [23, §4], Reeder and Yu connect the Moy-Prasad filtration at an epipelagic point with the Kac-Vinberg gradings of Lie algebras over the residue fields. We review their results here.

By the classification of hyperspecial points for split classical groups (see Remark in Appendix A.2), we can pick a point $x_{0} \in \mathcal{A}\left(\mathbf{T}, k^{\mathrm{ur}}\right)$ following the recipe in 23 , Section 3.2] such that $\operatorname{Jump}\left(\mathscr{L}_{E}^{0}\right)=\operatorname{Jump}\left(\mathscr{L}_{E}\right)$ where $\mathscr{L}_{E}^{0}$ is the lattice function in $V(E)$ corresponding to $x_{0}$. The action of the generator $\sigma$ of $\operatorname{Gal}\left(E / k^{\text {ur }}\right)$ on the Cartan subgroup $\mathbf{Y}$ induces an action $\vartheta$ on $X_{*}$. Then $x=x_{0}+\frac{1}{m} \check{\eta}$ where $\check{\eta} \in X_{*}^{\vartheta}$. Let $t:=\check{\eta}\left(\varpi_{E}\right) \in \mathbf{G}(E)$. The $\mathfrak{o}_{D(E)}$-lattice function corresponding to $x_{0}$ is $\mathscr{L}_{E}^{0}=t^{-1} \mathscr{L}_{E}$.

We have isomorphisms

$$
\mathbf{G}(E)_{x_{0}} \stackrel{\operatorname{Ad}(t)}{\longrightarrow} \mathbf{G}(E)_{x} \quad \text { and } \quad \mathfrak{g}(E)_{x_{0}, 0} \stackrel{\operatorname{Ad}(t)}{\longrightarrow} \mathfrak{g}(E)_{x, 0} \stackrel{\varpi_{E}^{j}}{\longrightarrow} \mathfrak{g}(E)_{x, \frac{j}{m}} .
$$

Let $\stackrel{\circ}{\mathbf{G}}=\mathbf{G}(E)_{x_{0}} / \mathbf{G}(E)_{x_{0}, 0^{+}}$and $\stackrel{\circ}{g}=\mathfrak{g}(E)_{x_{0}, 0} / \mathfrak{g}(E)_{x_{0}, 0^{+}}$. Let $\vartheta$ be the automorphisms on $\mathrm{G}(E)_{x_{0}}$ and $\mathrm{g}(E)_{x_{0}}$ induced by the $\sigma$ actions on $\mathbf{G}(E)_{x_{0}}$ and $\mathfrak{g}(E)_{x_{0}, 0}$ respectively. Let $\theta:=\operatorname{Ad}\left(t^{-1}\right) \circ \sigma \circ \operatorname{Ad}(t)$ be the automorphisms on $\mathrm{G}$ and on $\mathrm{g}$ induced by the $\sigma$ actions on $\mathbf{G}(E)_{x}$ and $\mathfrak{g}(E)_{x, 0}$. Let $\stackrel{\mathrm{g}}{\theta}, \zeta^{-j}$ be the $\zeta^{-j}$-eigenspace of $\theta$ on $\stackrel{\circ}{\mathrm{g}}$. Then

(a) $\theta=\operatorname{Ad}(\tilde{t}) \vartheta$ where $\tilde{t}=t^{-1} t^{\sigma}=\check{\eta}(\zeta)\left(\bmod G(E)_{x_{0}, 0^{+}}\right)$;

(b) $\operatorname{Ad}(t): \stackrel{\circ}{G}^{\theta} \stackrel{\sim}{\longrightarrow} \mathbf{G}_{x}:=\mathbf{G}\left(k^{\mathrm{ur}}\right)_{x} / \mathbf{G}\left(k^{\mathrm{ur}}\right)_{x, 0^{+}}$is an isomorphism and

(c) $\varpi_{E}^{j} \mathrm{ad}(t): \stackrel{\circ}{\mathrm{g}}^{\theta}, \zeta^{-j} \stackrel{\sim}{\longrightarrow} \mathrm{g}_{x, \frac{j}{m}}$ is $\dot{\mathrm{G}}^{\theta}$-equivariant with $\dot{\mathrm{G}}^{\theta}$ acting on the right hand side via (b). Here we recall $\mathrm{g}_{x, \frac{j}{m}}$ in Section 2.2.

By putting $j=-1$ in (c), we define $\iota_{\mathrm{g}}:=\varpi_{E}^{-1} \operatorname{ad}(t): \mathrm{g}^{\theta, \zeta} \sim \mathrm{g}_{x,-\frac{1}{m}}$. Then $\iota_{\mathrm{g}}$ is a bijection between the set of stable vectors for the $\dot{\mathrm{G}}^{\theta}$ action on $\stackrel{\circ}{\mathrm{g} \theta, \zeta}$ and the set of stable vectors for the action $\mathrm{G}\left(k^{\mathrm{ur}}\right)_{x}$ on $\mathrm{g}_{x,-\frac{1}{m}}$. The former was studied by Vinberg [26] and Levy [15].

\section{MOMEnT MAPS}


6.1. Let $W=V \otimes_{D} V^{\prime}$. Using the sesquilinear forms, we define $\Psi: W \stackrel{\sim}{\longrightarrow} \operatorname{Hom}_{D}\left(V, V^{\prime}\right)$ and $\Psi^{\prime}: W \stackrel{\sim}{\longrightarrow} \operatorname{Hom}_{D}\left(V^{\prime}, V\right)$ by

$$
\Psi\left(v \otimes v^{\prime}\right)\left(v_{1}\right)=v^{\prime}\left\langle v, v_{1}\right\rangle_{V} \text { and } \Psi^{\prime}\left(v \otimes v^{\prime}\right)\left(v_{1}^{\prime}\right)=v\left\langle v^{\prime}, v_{1}^{\prime}\right\rangle_{V^{\prime}}
$$

for all $v, v_{1} \in V$ and $v^{\prime}, v_{1}^{\prime} \in V^{\prime}$. Now $g \in G$ and $g^{\prime} \in G^{\prime}$ acts on $\operatorname{Hom}_{D}\left(V^{\prime}, V\right)$ by the formula $\left(g, g^{\prime}\right) \cdot w=g^{\prime} w g^{-1}$.

Definition 6.1.1. $\quad$ 1. We define a non-degenerate $G$-invariant symmetric $k$-bilinear form ${ }^{1} B_{\mathfrak{g}}: \mathfrak{g} \times \mathfrak{g} \rightarrow k$ by $B_{\mathfrak{g}}\left(X_{1}, X_{2}\right)=\frac{1}{2} \operatorname{tr}_{D / k} \operatorname{tr}\left(X_{2}^{*} X_{1}\right)$.

2. We define an operator $\star: \operatorname{Hom}_{D}\left(V, V^{\prime}\right) \rightarrow \operatorname{Hom}_{D}\left(V^{\prime}, V\right)$ by

$$
\left\langle w(v), v^{\prime}\right\rangle_{V^{\prime}}=\left\langle v, w^{\star}\left(v^{\prime}\right)\right\rangle_{V} \quad \forall w \in \operatorname{Hom}_{D}\left(V, V^{\prime}\right), v \in V, v^{\prime} \in V^{\prime} .
$$

We note that if $x \in W$ and $\Psi(x)=w$, then $\Psi^{\prime}(x)=w^{\star}$.

3. We define the moment map $M: W \cong \operatorname{Hom}_{D}\left(V, V^{\prime}\right) \rightarrow \mathfrak{g}$ and $M^{\prime}: W \rightarrow \mathfrak{g}^{\prime}$ by

$$
M(w)=w^{\star} w \text { and } M^{\prime}(w)=w w^{\star} .
$$

By definition $M$ and $M^{\prime}$ are $G \times G^{\prime}$-equivariant.

Lemma 6.1.2. Suppose $w_{1}, w_{2}, w \in W, X \in \mathfrak{g}$ and $X^{\prime} \in \mathfrak{g}^{\prime}$. Then

(a) $\left\langle w_{1}, w_{2}\right\rangle=\operatorname{tr}_{D / k} \operatorname{tr}\left(w_{2}^{\star} w_{1}\right)$,

(b) $\langle X \cdot w, w\rangle=2 B_{\mathfrak{g}}(M(w), X)$ and $\left\langle X^{\prime} \cdot w, w\right\rangle=2 B_{\mathfrak{g}^{\prime}}\left(-M^{\prime}(w), X^{\prime}\right)$.

The proof is a straightforward computation using (3) and the definition of $\star$. We will leave it to the reader.

6.2. Let $\mathscr{L}$ and $\mathscr{L}^{\prime}$ be two self-dual lattice functions on $V$ and $V^{\prime}$ respectively. Let $\mathscr{B}=\mathscr{L} \otimes \mathscr{L}^{\prime}$ on $W=V \otimes_{D} V^{\prime}$.

Lemma 6.2.1. (i) We have $\operatorname{Jump}(\mathscr{B})=\operatorname{Jump}(\mathscr{L})+\operatorname{Jump}\left(\mathscr{L}^{\prime}\right)$.

(ii) The lattice function $\mathscr{B}$ is self-dual in $W$, i.e. $\mathscr{B}_{r}^{\sharp}=\mathscr{B}_{-r^{+}}$.

(iii) Under the isomorphism $\Psi: W \sim \operatorname{Hom}_{D}\left(V, V^{\prime}\right)$,

$$
\Psi\left(\mathscr{B}_{r}\right)=\left\{w \in \operatorname{Hom}_{D}\left(V, V^{\prime}\right) \mid w \mathscr{L}_{s} \subseteq \mathscr{L}_{s+r}^{\prime} \forall s \in \mathrm{R}\right\} .
$$

(iv) We have $\left(\Psi\left(\mathscr{B}_{r}\right)\right)^{\star}=\Psi^{\prime}\left(\mathscr{B}_{r}\right)$.

(v) We have $M\left(\mathscr{B}_{r}\right) \subseteq \mathfrak{g}_{\mathscr{L}, 2 r}$ and $M^{\prime}\left(\mathscr{B}_{r}\right) \subseteq \mathfrak{g}_{\mathscr{L}^{\prime}, 2 r}^{\prime}$.

Proof. Part (i) is Equation (5). Part (ii) is explained in the Remark in Section 4.3. By (6) the right hand side of (iii) is the lattice function on $\operatorname{Hom}_{D}\left(V, V^{\prime}\right)=V^{*} \otimes_{D} V^{\prime}$. On the other hand $\Psi$ maps $V \otimes V^{\prime}$ to $V^{*} \otimes V^{\prime}$. We have seen in Section 4.3 that the norm $l^{*}$ on $V^{*}$ translates to the norm $l^{\sharp}=l$ on $V$. It follows that the lattice function on the right hand side of (iii) corresponds to the lattice function $\mathscr{B}$ under $\Psi$. This proves (iii). If $w \in W$ then $\Psi^{\prime}(w)=\Psi(w)^{\star}$. This proves (iv). Part (v) follows directly from (iii) and (iv).

6.3. We could view $\mathscr{B}_{s}, \mathfrak{g}_{\mathscr{L}, 2 s}$ and $\mathfrak{g}_{\mathscr{L}^{\prime}, 2 s}$ as schemes over $\mathfrak{o}_{k}$. Since $\star$ is $\mathfrak{o}_{k}$-linear, the moment maps defined over the generic fibers as in Section 6.1 extend to morphisms between these $\mathfrak{o}_{k}$-schemes. The $\mathfrak{o}_{k}$-group scheme $\mathbf{G}_{\mathscr{L}} \times \mathbf{G}_{\mathscr{L}^{\prime}}^{\prime}$ acts on all these objects and the moment maps are equivariant maps.

Let $\mathrm{W}_{s}=\mathscr{B}_{s} / \mathscr{B}_{s^{+}}, \mathrm{g}_{\mathscr{L}, 2 s}=\mathfrak{g}_{\mathscr{L}, 2 s} / \mathfrak{g}_{\mathscr{L}, 2 s^{+}}$and $\mathrm{g}_{\mathscr{L}^{\prime}, 2 s}^{\prime}=\mathfrak{g}_{\mathscr{L}^{\prime}, 2 s}^{\prime} / \mathfrak{g}_{\mathscr{L}^{\prime}, 2 s^{+}}^{\prime}$. We get morphisms, as certain quotients of the moment maps over the special fiber,

$$
\mathrm{M}_{s}: \mathrm{W}_{s} \rightarrow \mathrm{g}_{\mathscr{L}, 2 s} \text { and } \mathrm{M}_{s}^{\prime}: \mathrm{W}_{s} \rightarrow \mathrm{g}_{\mathscr{L}^{\prime}, 2 s}^{\prime} .
$$

The actions of $\mathbf{G}_{\mathscr{L}} \times \mathbf{G}_{\mathscr{L}^{\prime}}^{\prime}$ reduce to $\mathrm{G}_{\mathscr{L}} \times \mathrm{G}_{\mathscr{L}^{\prime}}^{\prime}$ actions on $\mathrm{W}_{s}, \mathrm{~g}_{\mathscr{L}, 2 s}$ and $\mathrm{g}_{\mathscr{L}^{\prime}, 2 s}^{\prime}$. These are the moment maps over the residual field $\mathfrak{f}$ which we will study later.

\footnotetext{
${ }^{1}$ We warn that our trace form $B_{\mathfrak{g}}$ has a factor of $\frac{1}{2}$.
} 


\section{THETA CORRESPONDENCES I}

7.1. In this section we let $\left(G, G^{\prime}\right)$ be a reductive dual pair in $\operatorname{Sp}(W)$ as in Section 3.2 . Let $\Sigma=(x, \lambda, \chi)$ and $\Sigma^{\prime}=\left(x^{\prime}, \lambda^{\prime}, \chi^{\prime}\right)$ be epipelagic supercuspidal data for $G$ and $G^{\prime}$ respectively. Let $\pi_{\Sigma}=\pi_{x}^{\widetilde{G}}(\lambda, \chi)$ (resp. $\pi_{\Sigma^{\prime}}^{\prime}=\pi_{x^{\prime}}^{\widetilde{G}^{\prime}}\left(\lambda^{\prime}, \chi^{\prime}\right)$ ) be the corresponding epipelagic representation of $\widetilde{G}$ (resp. $\left.\widetilde{G}^{\prime}\right)$. From now on, we assume $\theta\left(\pi_{\Sigma}\right)=\pi_{\Sigma^{\prime}}^{\prime}$. As discussed in Section 1.4, $x$ and $x^{\prime}$ are both epipelagic points of order $m$ for some $m \geq 2$.

Let $\mathscr{L}$ and $\mathscr{L}^{\prime}$ be self dual lattice functions corresponding to $x$ and $x^{\prime}$ respectively. Let $\mathscr{B}=\mathscr{L} \otimes \mathscr{L}^{\prime}$. Let $\mathrm{X}=\mathrm{W}_{-\frac{1}{2 m}}=\mathscr{B}_{-\frac{1}{2 m}} / \mathscr{B}_{-\frac{1}{2 m}}{ }^{+}$. We denote the moment maps defined in (8) by $\mathrm{M}: \mathrm{X} \rightarrow \mathrm{g}_{\mathscr{L},-\frac{1}{m}}$ and $\mathrm{M}^{\prime}: \mathrm{X} \rightarrow \mathrm{g}_{\mathscr{L}^{\prime},-\frac{1}{m}}^{\prime}$.

Proposition 7.1.1. Suppose that $\theta\left(\pi_{\Sigma}\right)=\pi_{\Sigma^{\prime}}^{\prime}$. Then there exists a $\bar{w} \in \mathbf{X}$ such that $\lambda=\mathrm{M}(\bar{w})$ and $\lambda^{\prime}=-\mathrm{M}^{\prime}(\bar{w})$.

Proof. By [22] there exists $\left(y, y^{\prime}\right) \in \mathcal{B}(\mathbf{G}, k) \times \mathcal{B}\left(\mathbf{G}^{\prime}, k\right), \mathscr{B}^{\prime}=\mathscr{L}_{y} \otimes \mathscr{L}_{y^{\prime}}^{\prime}$ and $w \in \mathscr{B}_{-\frac{1}{2 m}}^{\prime}$ such that $M(w)+\mathfrak{g}_{y,-\frac{1}{m}}{ }^{+}$is an unrefined minimal $K$-type of $\pi_{\Sigma}$, and $-M^{\prime}(w)+\mathfrak{g}_{y^{\prime},-\frac{1}{m}^{\prime}}{ }^{-}$ is an unrefined minimal $K^{\prime}$-type of $\pi_{\Sigma^{\prime}}^{\prime}$. The moment maps $M$ and $M^{\prime}$ commute with the action of $G \times G^{\prime}$-conjugation. By Proposition 2.3.2 and conjugating by $G \times G^{\prime}$, we may assume that $y=x, y^{\prime}=x^{\prime}, w \in \mathscr{B}_{-\frac{1}{2 m}}, M(w)+\mathfrak{g}_{x,-\frac{1}{m}}{ }^{+}=\mathbf{M}(\bar{w})=\lambda$ and $-M^{\prime}(w)+\mathfrak{g}_{x^{\prime},-\frac{1}{m}}^{\prime}{ }^{+}=-\mathrm{M}^{\prime}(\bar{w})=\lambda^{\prime}$ where $\bar{w}=w+\mathscr{B}_{-\frac{1}{2 m}}+\in \mathbf{X}$.

Corollary 7.1.2. We have $\operatorname{Jump}(\mathscr{B}) \subseteq \frac{1}{2 m}+\frac{1}{m} \mathrm{z}$. Moreover either

(i) $\operatorname{Jump}(\mathscr{L}) \subseteq \frac{1}{m} \mathrm{Z}$ and $\operatorname{Jump}\left(\mathscr{L}^{\prime}\right) \subseteq \frac{1}{2 m}+\frac{1}{m} \mathrm{Z}$ or

(ii) $\operatorname{Jump}(\mathscr{L}) \subseteq \frac{1}{2 m}+\frac{1}{m} \mathrm{Z}$ and $\operatorname{Jump}\left(\mathscr{L}^{\prime}\right) \subseteq \frac{1}{m} \mathrm{Z}$.

Proof. By Lemma 5.1.3, $\operatorname{Jump}(\mathscr{L})\left(\operatorname{resp.} \operatorname{Jump}\left(\mathscr{L}^{\prime}\right)\right)$ is a subset of $\frac{1}{m} \mathrm{z}$ or $\frac{1}{2 m}+\frac{1}{m} \mathrm{z}$. From the proof of the last proposition, $\bar{w}$ is a nonzero element in $\mathscr{B}_{-\frac{1}{2 m}} / \mathscr{B}_{-\frac{1}{2 m}}+$. In particular $-\frac{1}{2 m} \in \operatorname{Jump}(\mathscr{B})=\operatorname{Jump}(\mathscr{L})+\operatorname{Jump}\left(\mathscr{L}^{\prime}\right)$. The corollary follows.

7.2. Reeder and $\mathrm{Yu}$ connect the Moy-Prasad filtration at an epipelagic point with the Kac-Vinberg grading of Lie algebras over the residue fields, as described previously in Section 5.3. Now we relate this with the moment maps.

Let $\mathscr{L}_{E}^{0}=t^{-1} \mathscr{L}_{E}$ and $\mathscr{L}_{E}^{\prime 0}=t^{\prime-1} \mathscr{L}_{E}^{\prime}$ denote the $\mathfrak{o}_{D(E)}$-lattice functions corresponding to $x_{0}$ and $x_{0}^{\prime}$ respectively as in Section 5.2. By 7.1.2 we are in one of the following two cases.

(a) We have $\operatorname{Jump}\left(\mathscr{L}_{E}^{0}\right)=\frac{1}{m} \mathrm{z}$ and $\operatorname{Jump}\left(\mathscr{L}_{E}^{\prime 0}\right)=\frac{1}{2 m}+\frac{1}{m} \mathrm{Z}$. In this case we set $\mathrm{V}:=$ $\mathscr{L}_{E, 0: 0^{+}}^{0}$ and $\mathrm{V}^{\prime}:=\mathscr{L}_{E,-\frac{1}{2 m}:-\frac{1}{2 m}^{\prime 0}}{ }^{\text {. }}$

(b) We have $\operatorname{Jump}\left(\mathscr{L}_{E}^{0}\right)=\frac{1}{2 m}+\frac{1}{m} \mathrm{z}$ and $\operatorname{Jump}\left(\mathscr{L}_{E}^{\prime 0}\right)=\frac{1}{m} \mathrm{Z}$. In this case we set $\mathrm{V}:=$

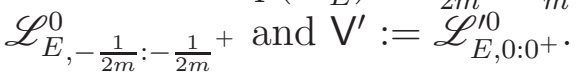

Both $\mathrm{V}$ and $\mathrm{V}^{\prime}$ are $\mathfrak{f}_{D(E)}$-modules. In Case (a), we assign a non-degenerate Hermitian forms $\vee$ by $\left\langle\langle,\rangle_{V}:=\langle,\rangle_{V}\left(\bmod \mathfrak{p}_{D(E)}\right)\right.$ and a non-degenerate Hermitian form on $\left\langle\langle,\rangle_{\mathrm{V}^{\prime}}:=\right.$ $\langle,\rangle_{V^{\prime}} \varpi_{E}\left(\bmod \mathfrak{p}_{D(E)}\right)$. In Case $(\mathrm{b})$, the bilinear forms are defined similarly. Thus $\stackrel{\circ}{\mathbf{G}}=$ $\mathrm{U}(\mathrm{V})$ (resp. $\stackrel{\circ}{\mathrm{G}}^{\prime}=\mathrm{U}\left(\mathrm{V}^{\prime}\right)$ ) as $\mathfrak{f}_{D(E)}$-linear transformations on $\mathbf{V}$ (resp. $\mathbf{V}^{\prime}$ ) preserving the form.

Let $\mathrm{L}^{0}(E)_{r}=\mathscr{L}_{E, r: r^{+}}^{0}$. The actions $\sigma$ and $t^{-1} \circ \sigma \circ t$ on $\mathscr{L}_{E, r}^{0}$ induce actions on $\mathrm{L}^{0}(E)_{r}$ which we denote by $\vartheta$ and $\theta$ respectively. It is compatible with the $\vartheta$ and $\theta$ actions on $\stackrel{\circ}{\mathrm{G}}$ defined in Section 5.3 in the sense that for $g \in \stackrel{\circ}{\mathrm{G}}$ we have $\vartheta(g)=\vartheta \circ g \circ \vartheta^{-1}$ and $\theta(g)=\theta \circ g \circ \theta^{-1}$ as linear transformations on $\mathrm{L}^{0}(E)_{r}$. 
Let $\mathscr{B}(E)=\mathscr{B} \otimes_{\mathfrak{o}_{k}} \mathscr{E}$ and $\mathscr{B}^{0}(E)=\left(t^{-1}, t^{\prime-1}\right) \mathscr{B}(E)=\mathscr{L}_{E}^{0} \otimes_{\mathfrak{o}_{D(E)}} \mathscr{L}_{E}^{\prime 0}$. Let $\mathrm{W}_{-\frac{1}{2 m}}^{0}=$ $\mathscr{B}^{0}(E)_{-\frac{1}{2 m}} / \mathscr{B}^{0}(E)_{-\frac{1}{2 m}}+$ and $\stackrel{\mathrm{W}}{\mathrm{W}}:=\mathrm{V} \otimes_{\mathfrak{f}_{D(E)}} \mathrm{V}^{\prime} \cong \operatorname{Hom}_{\mathfrak{f}_{D(E)}}\left(\mathrm{V}, \mathrm{V}^{\prime}\right)$. We observe the following diagram:

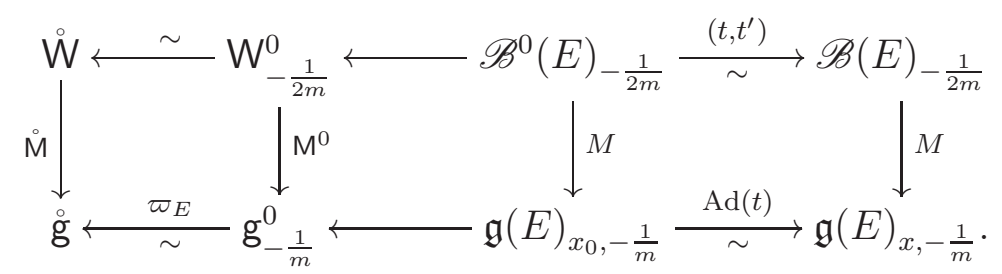

In the above diagram, the $\mathbf{G}(E)_{x} \times \mathbf{G}^{\prime}(E)_{x^{\prime}}$-equivariant map $M$ on the far right trans-

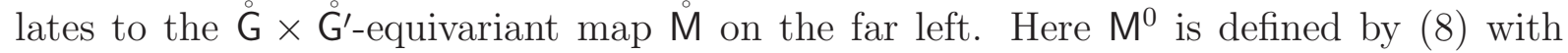
respect to lattices $\mathscr{L}_{E}^{0}$ and $\mathscr{L}_{E}^{\prime 0}$, and $s=-\frac{1}{2 m}$. The explicit formula for the map $\stackrel{\circ}{\mathrm{M}}$ is exactly the same as that for $M$ with respect to the forms $\langle\langle,\rangle\rangle_{v}$ and $\langle\langle,\rangle\rangle_{V^{\prime}}$. We remark that $W_{-\frac{1}{2 m}}^{0}$ is not equipped with any sesquilinear form. On the other hand $W^{\circ}$ has isomorphic vector space structure as $W_{-\frac{1}{2 m}}^{0}$ but it is equipped with a tensor product form.

7.3. The ranks of $G$ and $G^{\prime}$. Let $r$ be the rank of $\mathfrak{g}$ and let $P=P(X)$ be the coefficient of $z^{r}$ in $\operatorname{det}\left(z \mathrm{I}_{\mathfrak{g}}+\operatorname{ad} X\right)$. Then $P$ is an $\operatorname{Ad}(G)$-invariant homogeneous rational function on $\mathfrak{g}$ defined over $k$ such that the set of regular semisimple elements in $\mathfrak{g}$ is $P^{-1}(\mathrm{~A}-\{0\})$ where $\mathrm{A}$ is the affine line.

The following lemma is a consequence of [9, Lemma 13].

Lemma 7.3.1. Let $\lambda$ be a stable vector in $\mathfrak{g}_{x,-\frac{1}{m}:-\frac{1}{m}^{+}}$and $\gamma \in \mathfrak{g}_{x,-\frac{1}{m}}$ be a lifting of $\lambda$. Then $\gamma$ is a regular semisimple element. Let $\mathbf{T}=\mathrm{Z}_{\mathbf{G}}(\gamma)$. Then $\gamma$ is a good element of depth $-\frac{1}{2 m}$ with respect to $\mathbf{T}$, i.e. for every root $\alpha$ of $\mathbf{G}(\bar{k})$ with respect to $\mathbf{T}(\bar{k}), \mathrm{d} \alpha(\gamma)$ is nonzero and $\nu(\mathrm{d} \alpha(\gamma))=-\frac{1}{m}$.

Proof. Let $f(X)=\varpi_{E} \operatorname{Ad}(t)(X)$ and let $\bar{f}$ be the induced map in the following diagram:

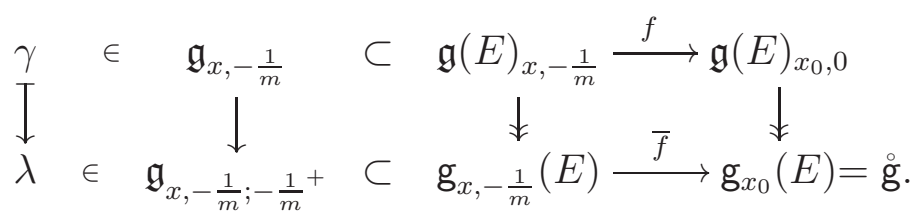

Since $\lambda$ is a stable vector, $\bar{f}(\lambda)$ is a regular semisimple element in $\stackrel{\circ}{\mathrm{g}}$ and $[P(f(\gamma))] \neq 0 \in \mathfrak{f}_{E}$. Therefore, $P(\gamma) \neq 0$ and $\gamma$ is a regular semisimple element in $\mathfrak{g}$.

Next we prove that $\gamma$ is good. Let $R(\mathbf{G}(\bar{k}), \mathbf{T}(\bar{k}))$ be the set of roots. Let $T^{\prime}(\bar{k}):=$ $\operatorname{Ad}(t) T(\bar{k})$. Then $\alpha \mapsto \alpha^{\prime}:=\alpha \circ \operatorname{Ad}\left(t^{-1}\right)$ gives a map $R(\mathbf{G}(\bar{k}), \mathbf{T}(\bar{k})) \rightarrow R\left(\mathbf{G}(\bar{k}), \mathbf{T}^{\prime}(\bar{k})\right)$. Moreover $\alpha^{\prime}$ reduces to a root $\overline{\alpha^{\prime}} \in \stackrel{\circ}{\mathrm{g}}$. For any $\alpha \in R(\mathbf{G}(\bar{k}), \mathbf{T}(\bar{k})), \overline{d \alpha^{\prime}}([f(\gamma)]) \neq 0$ since $[f(\gamma)]=\bar{f}(\lambda)$ is regular semisimple in $\stackrel{\circ}{\mathrm{g}}$. This implies that $\nu(\mathrm{d} \alpha(\gamma))=-\frac{1}{m}$ which proves the lemma.

Proposition 7.3.2. Suppose $\theta\left(\pi_{\Sigma}\right)=\pi_{\Sigma^{\prime}}^{\prime}$. Then $\left(\mathbf{G}, \mathbf{G}^{\prime}\right)$ or $\left(\mathbf{G}^{\prime}, \mathbf{G}\right)$ is one of the following types: (i) $\left(\mathrm{D}_{n}, \mathrm{C}_{n}\right)$, (ii) $\left(\mathrm{C}_{n}, \mathrm{D}_{n+1}\right)$, (iii) $\left(\mathrm{C}_{n}, \mathrm{~B}_{n}\right)$, (iv) $\left(\mathrm{A}_{n}, \mathrm{~A}_{n}\right)$, (v) $\left(\mathrm{A}_{n}, \mathrm{~A}_{n+1}\right)$.

Proof. By Proposition 7.1 .1 there exists $w \in \mathscr{B}_{-\frac{1}{2 m}} \subset W$ such that $[M(w)]=\mathrm{M}(\bar{w}) \in$ $\mathfrak{g}_{x,-\frac{1}{m}:-\frac{1}{m}}+$ and $\left[M^{\prime}(w)\right]=\mathrm{M}^{\prime}(\bar{w}) \in \mathfrak{g}_{x^{\prime},-\frac{1}{m}:-\frac{1}{m}}{ }^{+}$are stable vectors. By Lemma 7.3.1, both $M(w)$ and $M^{\prime}(w)$ are regular semisimple elements.

Now we show that (i) to (v) list all possible cases which satisfy the following condition.

(10) There is a $w \in W$ such that $M(w)$ and $M^{\prime}(w)$ are both regular semisimple. 
We may base change to the algebraic closure $\bar{k}$ so that $G=\mathbf{G}(\bar{k})$ and $G^{\prime}=\mathbf{G}^{\prime}(\bar{k})$ are split groups. We fix a maximal (split) torus $Y$ and identify its Lie algebra $\mathfrak{y}$ with $\AA^{\operatorname{dim} \mathfrak{y}}$. We list $\left.P\right|_{\mathfrak{y}}=\prod_{\alpha \in \Phi(G, Y)} d \alpha$ explicitly when $G$ is one the following groups.

$$
\begin{aligned}
& \mathrm{GL}(n): \mathfrak{y}=\mathrm{A}^{n}, P\left(a_{1}, \cdots, a_{n}\right)=\prod_{i \neq j}\left(a_{i}-a_{j}\right), \\
& \operatorname{Sp}(2 n): \mathfrak{y}=\mathrm{A}^{n}, P\left(a_{1}, \cdots, a_{n}\right)=\prod_{i \neq j}\left(a_{i}^{2}-a_{j}^{2}\right) \prod_{j}\left(-4 a_{j}^{2}\right), \\
& \mathrm{O}(2 n): \mathfrak{y}=\mathrm{A}^{n}, P\left(a_{1}, \cdots, a_{n}\right)=\prod_{i \neq j}\left(a_{i}^{2}-a_{j}^{2}\right), \\
& \mathrm{O}(2 n+1): \mathfrak{y}=\mathrm{A}^{n}, P\left(a_{1}, \cdots, a_{n}\right)=\prod_{i \neq j}\left(a_{i}^{2}-a_{j}^{2}\right) \prod_{j}\left(-a_{j}^{2}\right) .
\end{aligned}
$$

By the classification of dual pairs, we only have to consider one of the following reductive dual pairs:

(a) $\left(\mathrm{O}(2 n), \operatorname{Sp}\left(2 n^{\prime}\right)\right)$,

(b) $\left(\mathrm{Sp}(2 n), \mathrm{O}\left(2 n^{\prime}+1\right)\right)$ and

(c) $\left(\mathrm{GL}(n), \mathrm{GL}\left(n^{\prime}\right)\right)$.

We may assume that $\operatorname{rank} G \leq \operatorname{rank} G^{\prime}$. Let $\mathrm{W}$ and $\mathrm{W}^{\prime}$ be the Weyl groups of $G$ and $G^{\prime}$ with respect to $Y$ and $Y^{\prime}$ respectively. By the first and second fundamental theorems of classical invariant theory $M$ induces an isomorphism $W / G \cong \mathfrak{g}$ (see [7,11]) which in turn induces an isomorphism $W / G \times G^{\prime} \cong \mathfrak{g} / G^{\prime}$. We get following diagram:

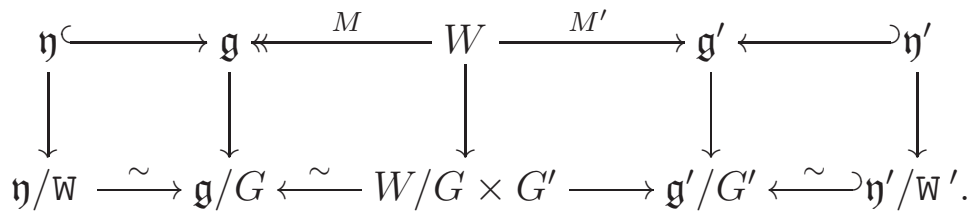

We remind the readers that in the lemma below all the vector spaces and algebraic groups are defined over $\bar{k}$.

Lemma 7.3.3. Suppose $\operatorname{rank} G \leq \operatorname{rank} G^{\prime}$. Then there is a vector subspace $\mathscr{A}$ of $W$ which is stable under the action of $Y \times Y^{\prime}$ and such that the following diagram below commutes.

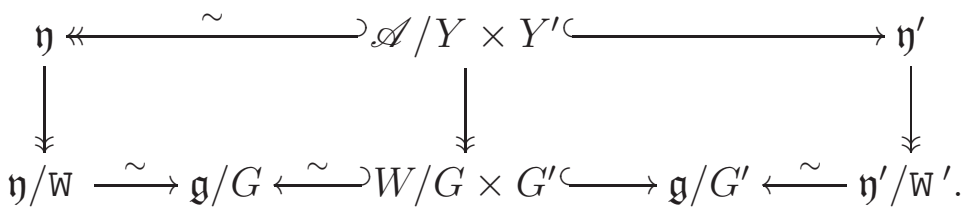

The proof of the lemma is given in Appendix B.1.

The top row of (11) defines an inclusion map $\Upsilon_{\mathfrak{y}}: \mathfrak{y} \hookrightarrow \mathfrak{y}^{\prime}$. This map is the natural inclusion $\mathrm{A}^{\operatorname{rank} G} \hookrightarrow \mathrm{A}^{\operatorname{rank} G^{\prime}}$ which is well known to the experts (for example see [1]).

Using the bottom row in (11), we have an inclusion $\mathfrak{y} / \mathfrak{W} \hookrightarrow \mathfrak{y}^{\prime} / \mathfrak{W}^{\prime}$ induced by $\Upsilon_{\mathfrak{y}}$. Let $P$ and $P^{\prime}$ be the invariant polynomials for $G$ and $G^{\prime}$ defined before Lemma 7.3.1. Then (10) is equivalent to the following statement:

There is an $X \in \mathfrak{y}$ such that $\left.P\right|_{\mathfrak{y}}(X) \neq 0$ and $\left.P^{\prime}\right|_{\mathfrak{y}^{\prime}}\left(\Upsilon_{\mathfrak{y}}(X)\right) \neq 0$.

It follows by inspection that (i) to (v) are all the possible cases.

\section{Theta CORRESPONDENCES II}

In this section we study theta correspondences of epipelagic representations. By Proposition 7.3.2, Proposition 7.1.1 and 7.1.2, it is enough to consider the following situations:

(C1) The dual pair $\left(\mathbf{G}, \mathbf{G}^{\prime}\right)$ is one of the following types: (i) $\left(\mathrm{D}_{n}, \mathrm{C}_{n}\right)$, (ii) $\left(\mathrm{C}_{n}, \mathrm{D}_{n+1}\right)$, (iii) $\left(\mathrm{C}_{n}, \mathrm{~B}_{n}\right)$, (iv) $\left(\mathrm{A}_{n}, \mathrm{~A}_{n}\right)$, (v) $\left(\mathrm{A}_{n}, \mathrm{~A}_{n+1}\right)$.

(C2) The points $x \in \mathcal{B}(\mathbf{G}, k)$ and $x^{\prime} \in \mathcal{B}\left(\mathbf{G}^{\prime}, k\right)$ are epipelagic points of order $m$. In particular, $m \geq 2$. Let $\mathscr{L}$ and $\mathscr{L}^{\prime}$ denote the corresponding $\mathfrak{o}_{D}$-lattice functions. 
(C3) $\mathscr{B}=\mathscr{L} \otimes \mathscr{L}^{\prime}$ where $\operatorname{Jump}(\mathscr{B}) \subseteq \frac{1}{2 m}+\frac{1}{m} \mathrm{Z}$.

(C4) There exists a $\bar{w} \in \mathrm{X}=\mathscr{B}_{-\frac{1}{2 m}} / \mathscr{B}_{\frac{1}{2 m}}$ such that $\mathrm{M}(\bar{w})=\lambda \in \mathrm{g}_{x,-\frac{1}{m}}$ and $-\mathrm{M}^{\prime}(\bar{w})=$ $\lambda^{\prime} \in \mathrm{g}_{x^{\prime},-\frac{1}{m}}^{\prime}$ are stable vectors.

8.1. We now study the geometry of $\mathbf{X}$ and the moment maps which will eventually determine the local theta correspondences.

By (C4) $\lambda$ is a stable vector so $\stackrel{\AA}{\lambda}:=\iota_{\mathrm{g}}^{-1}(\lambda)$ is a regular semisimple element in $\stackrel{\mathrm{g}}{\text { (c.f. }}$. Section 5.2). When $\lambda \in \stackrel{\mathrm{g}}{\subseteq} \operatorname{End}_{\mathfrak{f}_{D(E)}}(\mathrm{V})$ is not of full rank, it requires a special treatment. By (C1) and the classification of epipelagic points in [9, 23, this is exactly in the following situation:

$D$ is a ramified quadratic extension of $k$, the dual pair $\left(G, G^{\prime}\right)$ is a pair of unitary groups of the same rank $n$, and $\stackrel{\lambda}{\mathrm{\lambda}} \mathrm{g}$ has rank $n-1$.

Define

$$
\begin{aligned}
\mathrm{X}_{\lambda, \lambda^{\prime}} & :=\mathrm{M}^{-1}(\lambda) \cap \mathrm{M}^{\prime-1}\left(-\lambda^{\prime}\right) \text { and } \\
\mathrm{S}_{\bar{w}} & :=\operatorname{Stab}_{\mathrm{S}_{\lambda} \times \mathrm{S}_{\lambda^{\prime}}^{\prime}}(\bar{w}) .
\end{aligned}
$$

We note that $S_{\lambda}$ is abelian in all our cases so all its irreducible representations are one dimensional characters.

Lemma 8.1.1. (i) The set $\mathrm{X}_{\lambda, \lambda^{\prime}}$ is the $\mathrm{S}_{\lambda^{-}}$-orbit of $\bar{w}$ in $\mathrm{X}$.

(ii) There is a group homomorphism $\alpha: \mathrm{S}_{\lambda^{\prime}}^{\prime} \rightarrow \mathrm{S}_{\lambda}$ such that $\triangle_{\alpha}\left(\mathrm{S}_{\lambda^{\prime}}^{\prime}\right):=\left\{\left(\alpha\left(g^{\prime}\right), g^{\prime}\right) \mid g^{\prime} \in \mathrm{S}_{\lambda^{\prime}}^{\prime}\right\}$ is a subgroup of $\mathrm{S}_{\bar{w}}$.

(iii) If we are not in Case (E), then $\mathrm{S}_{\lambda}$ acts freely on $\mathrm{X}_{\lambda, \lambda^{\prime}}$ and $\mathrm{S}_{\bar{w}}=\triangle_{\alpha}\left(\mathrm{S}_{\lambda^{\prime}}^{\prime}\right)$.

(iv) In Case (E), let $\mathrm{S}_{\bar{w}}=\operatorname{Stab}_{\mathrm{S}_{\lambda}}(\bar{w})$ and $\mathrm{S}_{\lambda}:=\left\{g \in \mathrm{S}_{\lambda} \mid g \circ \lambda=\lambda\right\}$. Then $\mathrm{S}_{\bar{w}}=\mathrm{S}_{\lambda}$ and so the character $\chi$ of $\mathrm{S}_{\lambda}$ occurs in $\mathrm{C}\left[\mathrm{X}_{\lambda, \lambda^{\prime}}\right]$ if and only if $\left.\chi\right|_{\mathrm{S}_{\lambda}}$ is trivial.

The proof is given in Appendix B.2.

Remark. (1) The homomorphism $\alpha$ induces a map $\alpha^{*}: \widehat{\mathrm{S}_{\lambda}} \rightarrow \widehat{\mathrm{S}_{\lambda^{\prime}}}$ given by $\alpha^{*}(\chi)=\chi \circ \alpha$. The definition depends on the choice of $\bar{w} \in \mathbf{X}_{\lambda, \lambda^{\prime}}$. On the other hand, it is well-defined up to conjugation by the proof in Appendix B.2. Hence the map between the Grothendieck groups induced by $\alpha^{*}$ is independent of the choice of $\bar{w}$.

(2) In the exceptional Case (E), Lemma 8.1.1 (iv) will lead to the fact that not all epipelagic representations can occur in this local theta correspondence. The extreme case is the well known fact that not all characters of $\mathrm{U}(1)$ occur in the oscillator representation of $\operatorname{Mp}(2)($ see [18]).

Theorem 8.1.2. Suppose (C1) to (C4) hold. For any character $\chi$ of $\mathrm{S}_{\lambda}$, let $\Sigma=(x, \lambda, \chi)$ and $\Sigma^{\prime}=\left(x^{\prime}, \lambda^{\prime}, \chi^{\prime}\right)$ where $\chi^{\prime}=\chi^{*} \circ \alpha$ and $\chi^{*}$ is the contragredient representation of $\chi$.

(i) Suppose we are not in the exceptional case (E). Then

$$
\theta\left(\pi_{\Sigma}\right)=\pi_{\Sigma^{\prime}}^{\prime}
$$

In particular the theta lift is nonzero.

(ii) Suppose we are in the exceptional Case (E). Then (12) holds for $\chi \in \widehat{\mathrm{S}_{\lambda}}$ such that $\left.\chi\right|_{\mathrm{s}_{\lambda}}$ is trivial.

The above theorem gives 1.4 .3 (i).

\footnotetext{
${ }^{2}$ Let $h \in G_{x}$ and $\gamma \in \mathfrak{g}_{x,-\frac{1}{m}}$ be any lifts of $g$ and $\lambda$ respectively. We consider $h$ and $\gamma$ as elements in $\operatorname{Hom}_{k}(V, V)$. Then $g \circ \lambda:=h \circ \gamma+\mathfrak{g}_{x,-\frac{1}{m}}+\in \mathfrak{g}_{x,-\frac{1}{m}:-\frac{1}{m}}+$ is well defined.
} 
8.2. We set $B=\mathscr{B}_{-\frac{1}{2 m}}$ and $A=\mathscr{B}_{\frac{1}{2 m}}$. By (C3), $A^{\sharp}=A$ and $B^{\sharp}=\mathscr{B}_{\frac{1}{2 m}}{ }^{+}=\mathscr{B}_{\frac{3}{2 m}}$. Since $m \geq 2, \mathscr{B}_{-\frac{1}{2 m}} \mathfrak{p}_{k} \subseteq \mathscr{B}_{\frac{3}{2 m}}$ and $B^{\sharp}$ is a good lattice. We form the following exact sequences of $\mathfrak{f}$-vector spaces:

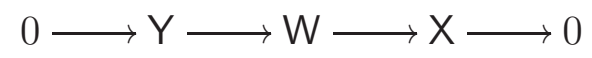

where

$$
\mathrm{W}=B / B^{\sharp}=\mathscr{B}_{-\frac{1}{2 m}: \frac{3}{2 m}}, \quad \mathrm{X}=B / A=\mathscr{B}_{-\frac{1}{2 m}: \frac{1}{2 m}} \text { and } \mathrm{Y}=A / B^{\sharp}=\mathscr{B}_{\frac{1}{2 m}: \frac{3}{2 m}} .
$$

The symplectic form on $W$ induces a non-degenerate $\mathfrak{f}$-symplectic form on $\mathrm{W}$ and $\mathrm{Y}$ is a maximal isotropic subspace in W. Let

$$
\begin{aligned}
& \mathrm{P}=\left(G_{\mathscr{L}} / G_{\mathscr{L}, \frac{2}{m}}\right) \times\left(G_{\mathscr{L}^{\prime}}^{\prime} / G_{\mathscr{L}^{\prime}, \frac{2}{m}}^{\prime}\right) \text { and } \\
& \mathrm{J}=\left(G_{\mathscr{L}} / G_{\mathscr{L}, \frac{1}{m}}\right) \times\left(G_{\mathscr{L}^{\prime}}^{\prime} / G_{\mathscr{L}^{\prime}, \frac{1}{m}}^{\prime}\right)=\mathrm{G}_{x} \times \mathrm{G}_{x^{\prime}}^{\prime} .
\end{aligned}
$$

Then (13) is an exact sequence of P-modules. The proof of following lemma is given in Appendix A.

Lemma 8.2.1. The natural quotient $\mathrm{P} \rightarrow \mathrm{J}$ has a splitting such that the exact sequence (13) splits as J-modules. We denote the splitting by $\mathrm{W}=\mathrm{Y} \oplus \mathrm{X}$.

8.3. Proof of 8.1.2. We recall the lattice model $\mathscr{S}(A)$ in Section 3.3. Let $\mathscr{S}(A)_{B}$ be the subspace of functions in $\mathscr{S}(A)$ with support in $B$. For $f \in \mathscr{S}(A)_{B}, w \in B$ and $b^{\prime} \in B^{\sharp}, f\left(w+b^{\prime}\right)=\psi\left(\frac{1}{2}\left\langle w, b^{\prime}\right\rangle\right) f(w)=f(w)$. Therefore, we could view $\mathscr{S}(A)_{B}$ as a subspace in $\mathrm{C}[\mathrm{W}]$. We fix the splitting $\mathrm{W}=\mathrm{Y} \oplus \mathrm{X}$ in Lemma 8.2.1. Let $\mathrm{J}$ act on $\mathrm{C}[\mathrm{X}]$ by translation. Since $f(w+a)=\psi\left(\frac{1}{2}\langle w, a\rangle\right) f(w)$ for all $a \in A$, the restriction map $R_{\mathrm{X}}$ from $\mathrm{W}$ to $\mathrm{X}$ induces a $\mathrm{J}$-module isomorphism

$$
R_{\mathrm{X}}: \mathscr{S}(A)_{B} \stackrel{\sim}{\longrightarrow} \mathrm{C}[\mathrm{X}]
$$

whose inverse map $R_{\mathrm{x}}^{-1}$ is given by

$$
\left(R_{\mathrm{X}}^{-1} F\right)(w)=\psi\left(\frac{1}{2}\langle x, y\rangle\right) F(x)
$$

for all $w \in B$ such that $w \equiv x+y\left(\bmod B^{\sharp}\right)$ with $x+B^{\sharp} \in \mathrm{X}$ and $y+B^{\sharp} \in \mathrm{Y}$.

For $f \in \mathscr{S}(A)_{B}, w \in B$ and $g \in G_{\mathscr{L}, \frac{1}{m}}$, we have $\left(g^{-1}-1\right) w \in A$. By (44) and Lemma 6.1.2, we have 3

$$
\begin{aligned}
\omega_{A}(g) f(w) & =f\left(g^{-1} w\right)=f\left(\left(g^{-1}-1\right) w+w\right) \\
& =\psi\left(\frac{1}{2}\left\langle w,\left(g^{-1}-1\right) w\right\rangle\right) f(w)=\psi\left(\frac{1}{2}\langle(g-1) w, w\rangle\right) f(w) \\
& =\psi\left(B_{\mathfrak{g}}(M(w), c(g))\right) f(w)=\psi_{\mathrm{M}(\bar{w})}(g) f(w)
\end{aligned}
$$

where $\bar{w}$ is the image of $w$ in $\mathbf{X}=B / A$. Similarly $\omega_{A}\left(g^{\prime}\right) f(w)=\psi_{-\mathbf{M}^{\prime}(\bar{w})}\left(g^{\prime}\right) f(w)$ for all $g^{\prime} \in G_{\mathscr{L}, \frac{1}{m}}$

Let $\mathscr{S}(A)_{B}^{\lambda}$ be the subspace of functions in $\mathscr{S}(A)_{B}$ such that $G_{\mathscr{L}, \frac{1}{m}}$ acts by $\psi_{\lambda}$. Then it follows from (15) and (14) that

$$
\mathscr{S}(A)_{B}^{\lambda}=R_{\mathrm{X}}^{-1}\left(\mathrm{C}\left[\mathrm{M}^{-1}(\lambda)\right]\right) .
$$

A similar consideration applies to $\lambda^{\prime} \in \mathrm{g}_{\mathscr{L}^{\prime},-\frac{1}{m}}^{\prime}$ too. Let $\mathscr{S}^{\lambda, \lambda^{\prime}}:=\mathscr{S}(A)_{B}^{\lambda} \cap \mathscr{S}(A)_{B}^{\lambda^{\prime}}$. Then

$$
\mathscr{S}^{\lambda, \lambda^{\prime}}=R_{\mathrm{X}}^{-1}\left(\mathrm{C}\left[\mathrm{X}_{\lambda, \lambda^{\prime}}\right]\right) \text {. }
$$

\footnotetext{
${ }^{3}$ See also the proof of [22, Theorem 5.5]
} 
We fix a $\bar{w}$ in $\mathrm{X}_{\lambda, \lambda^{\prime}}$. By Lemma 8.1.1, $\mathrm{S}_{\lambda} \rightarrow \mathrm{X}_{\lambda, \lambda^{\prime}}$ given by $s \mapsto s \cdot \bar{w}$ is a surjection of $\mathrm{S}_{\lambda} \times \mathrm{S}_{\lambda^{\prime}}^{\prime}$-set. Here $\left(s, s^{\prime}\right) \in \mathrm{S}_{\lambda} \times \mathrm{S}_{\lambda^{\prime}}^{\prime}$ acts on $\mathrm{S}_{\lambda}$ by $\left(s, s^{\prime}\right) \cdot s_{0}=s s_{0} \alpha\left(s^{\prime}\right)^{-1}$ for all $s_{0} \in \mathbf{S}_{\lambda}$.

By the decomposition of regular representation of $S_{\lambda}$, we have

$$
\mathrm{c}\left[\mathrm{X}_{\lambda, \lambda^{\prime}}\right] \subseteq \mathrm{C}\left[\mathrm{S}_{\lambda}\right]=\bigoplus_{\chi \in \widehat{\mathrm{S}_{\lambda}}} \mathrm{C}_{\chi} \otimes \mathrm{C}_{\chi^{*} \circ \alpha}
$$

as $\mathrm{S}_{\lambda} \times \mathrm{S}_{\lambda^{\prime}}^{\prime}$-modules. In (i), (16) is an equality. In (ii) the summand $\mathrm{C}_{\chi} \otimes \mathrm{C}_{\chi^{*} \circ \alpha}$ occurs in $\mathrm{C}\left[\mathrm{X}_{\lambda, \lambda^{\prime}}\right]$ if and only if $\left.\chi\right|_{S_{\lambda}}$ is trivial by Lemma 8.1.1.

Fix any $\mathrm{S}_{\lambda^{-}}$-character $\chi$ which occurs in $\mathrm{C}\left[\mathrm{X}_{\lambda, \lambda^{\prime}}\right]$. It is clear that the $H_{\lambda}$ and $H_{\lambda^{\prime}}^{\prime}$ in (1) act on the space $R_{\mathrm{X}}^{-1}\left(\mathrm{C}_{\lambda} \otimes \mathrm{C}_{\chi^{*} \circ \alpha}\right)$ by the characters $\psi_{\lambda} \otimes \chi$ and $\psi_{\lambda^{\prime}} \otimes \chi^{*} \circ \alpha$ respectively. By Frobenius reciprocity, the subspace $R_{\mathrm{x}}^{-1}\left(\mathrm{C}_{\lambda} \otimes \mathrm{C}_{\chi^{*} \circ \alpha}\right) \subseteq \mathscr{S}(A)$ induces a non-zero intertwining map

$$
\pi_{x}^{\widetilde{G}}(\lambda, \chi) \otimes \pi_{x^{\prime}}^{\widetilde{G}^{\prime}}\left(\lambda^{\prime}, \chi^{*} \circ \alpha\right) \longrightarrow \mathscr{S}(A) .
$$

The left hand side is irreducible so the above map is an injection. Since the left hand side is also supercuspidal, by the smoothness of $\mathscr{S}(A)$, we conclude that the left hand side is a direct summand in $\mathscr{S}(A)$ and we have a projection map from $\mathscr{S}(A)$ to the left hand side. This completes the proof of 8.1.2.

8.4. Proof of 1.4.3 (ii). Part (a) is a restatement of Proposition 7.1.1. By 1.4.3 $\pi_{\Sigma}$ has a nonzero theta lift and $\theta\left(\pi_{\Sigma}\right)=\pi_{\Sigma^{\prime \prime}}^{\prime}$ where $\Sigma^{\prime \prime}=\left(x^{\prime}, \lambda^{\prime}, \chi^{*} \circ \alpha\right)$. By the uniqueness of the theta lift [6, 27], $\pi_{\Sigma^{\prime}}^{\prime}=\pi_{\Sigma^{\prime \prime}}^{\prime}$ and by Proposition 2.3.2 $\chi^{\prime}=\chi^{*} \circ \alpha$. This proves (b).

\section{Appendix A. Proof of Lemma 8.2.1}

A.1. First the fact that $\mathrm{P} \rightarrow \mathrm{J}$ splits follows from the work of McNinch [16]. For our case, the splitting could be constructed by an elementary method which we will explain below.

We retain the notation in Section 3.1. Let $K$ be the maximal unramified extension in $D$ defined in the following way: (i) $K:=k$ if $D=k$; (ii) $K:=D$ if $D / k$ is an unramified extension; (iii) $K:=k$ if $D / k$ is a ramified extension and (iv) if $D$ the quaternion algebra over $k$, then $K$ is the unramified quadratic extension of $k$ in $D$ normalized by $\varpi_{D}$.

Let $\nu_{D}=\nu\left(\varpi_{D}\right)$. Then $\nu_{D}=\frac{1}{2}$ if and only if $D / k$ is ramified or $D$ is the quaternion algebra over $k$. Under this setting, $D=K$ if $\nu_{D}=1$ and $D=K \oplus \varpi_{D} K$ if $\nu_{D}=\frac{1}{2}$. In all cases, $\mathfrak{f}_{K}=\mathfrak{f}_{D}$.

A.2. We recall the explicit description of an apartment in $\mathcal{B}(\mathbf{G}, k)$ (c.f. [5, §2.9] and [3, §2-4]). Let $\lceil r\rceil$ denote the largest integer not greater than $r \in \mathrm{R}$. Let $n$ be the dimension of a maximally isotropic subspace in $V$. Let $I:=I^{+} \sqcup I^{-} \sqcup I^{0}$ where $I^{+}=\{1, \cdots, n\}$, $I^{-}=-I^{+}$and $I^{0}$ is any index set with $\operatorname{dim}_{D} V-2 n$ elements. Fix a basis $\left\{e_{i} \mid i \in I\right\}$ of $V$ such that

(a) $\left\langle e_{i}, e_{j}\right\rangle_{V}=\left\langle e_{-i}, e_{-j}\right\rangle_{V}=0$ and $\left\langle e_{i}, e_{-j}\right\rangle_{V}=\delta_{i, j}$ for all $i, j \in I^{+}$;

(b) $e_{i}$ is anisotropic for $i \in I^{0}$ and $\left\langle e_{i}, e_{j}\right\rangle_{V}=0$ for $i \in I^{0}$ and $i \neq j \in I$.

For $i \in I^{0}$, we can choose $e_{i}$ such that

(i) $\left\langle e_{i}, e_{i}\right\rangle_{V}$ has valuation either 0 or $\nu_{D}$;

(ii) $\left\langle e_{i}, e_{i}\right\rangle_{V}$ takes value either in $\mathfrak{o}_{K}$ or in $\varpi_{D} \mathfrak{o}_{K}$. U

Let $\mathbf{S}$ be the maximal $k$-split torus in $\mathbf{G}$ which stabilizes $e_{i} D$ for all $i \in I^{+} \sqcup I^{-}$and fixes $e_{j}$ for all $j \in I^{0}$. Then the apartment $\mathcal{A}(\mathbf{S}, k)$ in $\mathcal{B}(\mathbf{G}, k)$ corresponds to the set

\footnotetext{
${ }^{4}$ This condition is non-trivial if $D / k$ is a ramified extension or $D / k$ is a quaternion algebra.
} 
of self-dual lattice functions which split under this basis. More precisely, if $\mathscr{L}$ is in the apartment, then there is a (unique) tuple of real numbers $\left(a_{1}, \cdots, a_{n}\right) \in \mathrm{R}^{n}$ such that

$$
\mathscr{L}_{r}=\bigoplus_{i \in I} e_{i} \mathfrak{p}_{D}^{\left\lceil\left(r-a_{i}\right) / \nu_{D}\right\rceil}
$$

where (i) $a_{-i}=-a_{i}$ for $i \in I^{+}$and (ii) $a_{i}=\frac{1}{2} \nu\left(\left\langle e_{i}, e_{i}\right\rangle_{V}\right)$. In fact, $\left(a_{1}, \cdots, a_{n}\right) \mapsto \mathscr{L}$ gives an identification of $\mathrm{R}^{n}$ with the apartment.

Remark. If $\mathbf{G}$ splits over an unramified extension of $k$, then the lattice function in (17) corresponds to a hyperspecial point in $\mathcal{A}(\mathbf{S}, k)$ if and only if $a_{i} / \nu_{D} \in b+\mathrm{z}$ for all $i \in I$ where $b=0$ or $\frac{1}{2}$.

A.3. We let $\mathscr{L}$ be a lattice function as in (17) above. We consider two cases.

Case 1. First we assume that $\nu_{D}=1$. In this case $D=K$. Let $[r]$ denote the coset $r+\mathrm{Z} \in \mathrm{Q} / \mathrm{Z}$ and define

$$
V^{[r]}:=\sum_{a_{i} \equiv r} e_{(\bmod \mathrm{z})} K \quad \text { and } \quad \mathscr{V}^{r}:=V^{[r]} \cap \mathscr{L}_{r}=\sum_{a_{i} \equiv r} e_{(\bmod \mathrm{z})} e_{i} \mathfrak{p}_{K}^{r-a_{i}} .
$$

We make the following observations.

(a) The restriction of the Hermitian sesquilinear form to $V^{[r]}$ is non-degenerate if $r \equiv 0$ or $\frac{1}{2}(\bmod z)$ and totally isotropic if otherwise.

(b) For $r \in \mathrm{R}, V^{[r]}$ is in perfect pairing with $V^{[-r]}$. In particular, $\mathscr{V}^{0}$ and $\mathscr{V}^{\frac{1}{2}}$ have $\epsilon$-Hermitian sesquilinear forms $\langle,\rangle_{V}$ and $\langle,\rangle_{V} \varpi_{D}^{-1}$ which are defined over $\mathfrak{o}_{K}$.

Case 2. Now assume $\nu_{D}=\frac{1}{2}$. We define the $K$-module

$$
V^{[r]}:=\sum_{a_{i} \equiv r} e_{i} K+\sum_{a_{i}+\frac{1}{2} \equiv r} e_{i} \varpi_{D} K
$$

and $\mathfrak{o}_{K}$-module

$$
\mathscr{V}^{r}:=V^{[r]} \cap \mathscr{L}_{r}=\sum_{a_{i} \equiv r} e_{i} \mathfrak{p}_{K}^{r-a_{i}}+\sum_{a_{i}+\frac{1}{2} \equiv r} e_{i} \varpi_{D} \mathfrak{p}_{K}^{r-a_{i}-\frac{1}{2}} .
$$

We make the following observations.

(a) The two $K$-subspaces $V^{[r]}$ and $V^{\left[r+\frac{1}{2}\right]}$ in $V$ are different. However $V^{[r]}=V^{\left[r+\frac{1}{2}\right]} \varpi_{D}$ and $\mathscr{V}^{r+\frac{1}{2}}=\mathscr{V}^{r} \varpi_{D}$

(b) The restriction of the Hermitian sesquilinear form to $V^{[r]}$ is non-degenerate if $r \equiv 0$ or $\frac{1}{4}\left(\bmod \frac{1}{2} z\right)$ and totally isotropic if otherwise.

(c) For $r \in \mathrm{R}, V^{[r]}$ is in perfect pairing with $V^{[-r]}$. In particular, there is an $\epsilon$-Hermitian sesquilinear form $\langle,\rangle_{V}$ and a $(-\epsilon)$-Hermitian sesquilinear form $\langle,\rangle_{V} \varpi_{D}^{-1}$ defined on $\mathscr{V}^{0}$ and $\mathscr{V}^{\frac{1}{4}}$ respectively. Both forms are defined over $\mathfrak{o}_{K}$.

Thanks to the definitions of $V^{[r]}$ and $\mathscr{V}^{r}$, the following holds for both Cases 1 and 2:

(i) $\operatorname{dim}_{K} V^{[r]}=\operatorname{dim}_{\mathfrak{f}_{D}} \mathscr{L}_{r} / \mathscr{L}_{r^{+}}$and the natural inclusion $\mathscr{V}^{r} \hookrightarrow \mathscr{L}_{r}$ induces an isomorphism

$$
\mathscr{V}^{r} / \mathscr{V}^{r} \mathfrak{p}_{K} \stackrel{\sim}{\longrightarrow} \mathscr{L}_{r} / \mathscr{L}_{r^{+}}
$$

(ii) $V=\bigoplus_{[r] \in Q / z} V^{[r]}$.

(iii) Define an $\mathfrak{o}_{k}$-group scheme:

$$
\mathscr{Q}:=\mathrm{U}\left(\mathscr{V}^{0}\right) \times \mathrm{U}\left(\mathscr{V}^{\frac{1}{2} \nu_{D}}\right) \times \prod_{r \in\left(0, \frac{1}{2} \nu_{D}\right)} \mathrm{GL}_{\mathfrak{o}_{K}}\left(\mathscr{V}^{r}\right) .
$$


Let $Q$ and $\mathrm{Q}$ denote the generic fiber and special fiber of $\mathscr{Q}$ respectively.

(iv) The lattices $\mathscr{V}^{r}$ give a vertex $y$ in the building of $Q$. Clearly,

$$
\mathscr{Q}=Q_{y}, \quad Q_{y, 0^{+}}=Q_{y, 1} \quad \text { and } \quad \mathrm{Q}=Q_{y} / Q_{y, 0^{+}} .
$$

(v) The natural action of $\mathscr{Q}$ on $V$ identifies $Q$ with $G \cap\left(\prod_{[r] \in Q / z} G_{K}\left(V^{[r]}\right)\right)$ so that $\mathscr{Q}=Q \cap G_{\mathscr{L}}$.

(vi) The natural embedding $\mathscr{Q} \rightarrow G_{\mathscr{L}}$ induces an isomorphism of $\mathfrak{f}$-groups

$$
\mathrm{Q}=Q_{y} / Q_{y, 1} \stackrel{\sim}{\longrightarrow} G_{\mathscr{L}} / G_{\mathscr{L}, 0^{+}}=\mathrm{G}_{\mathscr{L}}
$$

which is compatible with (18). This follows for the fact that the both sides are isomorphic to the right hand side of (7).

For $V^{\prime}$, we likewise divide into two cases and define similar notations $V^{\prime[r]}, \mathscr{V}^{\prime r}, Q^{\prime}, \mathscr{Q}^{\prime}$ etc as above.

A.4. We recall $\mathscr{B}=\mathscr{L} \otimes \mathscr{L}^{\prime}$. For $\mu \in \mathrm{R}$, we define $X^{[\mu]}=\sum_{[t]+\left[t^{\prime}\right]=[\mu]} V^{[t]} \otimes_{K} V^{\left[t^{\prime}\right]}$. Then

(vii) $W=\bigoplus_{[\mu] \in Q / \mathrm{z}} X^{[\mu]}$

(viii) $\mathscr{X}_{\mu}:=\sum_{t+t^{\prime}=\mu} \mathscr{V}^{t} \otimes_{\mathfrak{o}_{K}} \mathscr{V}^{\prime t^{\prime}}$ equals $X^{[\mu]} \cap \mathscr{B}_{\mu}$.

Using the natural inclusion $\mathscr{X}_{\mu} \hookrightarrow \mathscr{B}_{\mu}$, we have

(ix) $\mathscr{X}_{\mu+1}=\mathscr{X}_{\mu^{+}}:=X_{[\mu]} \cap \mathscr{B}_{\mu^{+}}$.

(x) $\mathscr{X}_{\mu} / \mathscr{X}_{\mu+1} \stackrel{\sim}{\longrightarrow} \mathscr{X}_{\mu} / \mathscr{X}_{\mu^{+}} \stackrel{\sim}{\longrightarrow} \mathscr{B}_{\mu} / \mathscr{B}_{\mu^{+}}$.

Proof of Lemma 8.2.1. We recall (13) where

$$
\mathrm{Y}:=\mathscr{B}_{\frac{1}{2 m}: \frac{1}{2 m}}+, \quad \mathrm{W}:=\mathscr{B}_{-\frac{1}{2 m}: \frac{1}{2 m}}{ }^{+} \quad \text { and } \quad \mathrm{X}:=\mathscr{B}_{-\frac{1}{2 m}:-\frac{1}{2 m}}+.
$$

Let $\mathrm{X}^{\prime}:=\mathscr{X}_{-\frac{1}{2 m}} / \mathscr{X}_{-\frac{1}{2 m}} \mathfrak{p}_{K}$. Clearly $\mathrm{X}^{\prime} \stackrel{\sim}{\longrightarrow} \mathrm{X}$ by $(\underline{\mathrm{X}})$.

Note that $m \geq 2$. So $\frac{1}{m}<1$. The inclusion $\mathscr{X}_{-\frac{1}{2 m}} \hookrightarrow \mathscr{B}_{-\frac{1}{2 m}}$ gives an embedding

$$
\mathrm{X}^{\prime}=\mathscr{X}_{-\frac{1}{2 m}} / \mathscr{X}_{-\frac{1}{2 m}+1} \longrightarrow \mathscr{B}_{-\frac{1}{2 m}} / \mathscr{B}_{\frac{1}{2 m}}{ }^{+}=\mathrm{W}
$$

which splits the quotient map $\mathrm{W} \rightarrow \mathrm{X}$. The embedding $\mathscr{Q} \times \mathscr{Q}^{\prime} \hookrightarrow G_{\mathscr{L}} \times G_{\mathscr{L}^{\prime}}^{\prime}$ induces a splitting of $\mathrm{P} \rightarrow \mathrm{J}$ :

$$
\mathrm{J} \longleftarrow \mathrm{Q} \times \mathrm{Q}^{\prime}=\mathscr{Q} / \mathscr{Q}_{1} \times \mathscr{Q}^{\prime} / \mathscr{Q}_{1}^{\prime} \longrightarrow G_{\mathscr{L}} / G_{\mathscr{L}, \frac{1}{m}}{ }^{+} \times G_{\mathscr{L}^{\prime}}^{\prime} / G_{\mathscr{L}^{\prime}, \frac{1}{m}}^{\prime}{ }^{+}=\mathrm{P} .
$$

Note that $\mathrm{Y}, \mathrm{X}^{\prime}$ and $\mathrm{W}$ are natural $\mathrm{Q} \times \mathrm{Q}^{\prime}$-modules and (19) is an $\mathrm{Q} \times \mathrm{Q}^{\prime}$-equivariant embedding. We get a decomposition $\mathrm{W}=\mathrm{X}^{\prime} \oplus \mathrm{Y}$ as J-modules under the splitting (20).

\section{Appendix B. Matrix CALCUlations}

In this appendix, we prove Lemma 7.3.3 and Lemma 8.1.1.

B.1. Proof of Lemma 7.3.3. We construct below an $\mathscr{A}$ defined over an algebraically closed field $\bar{k}$ which satisfies the lemma. The lemma and the proof below is valid for any field provided $\left(G, G^{\prime}\right)$ is an irreducible dual pair such that $G$ and $G^{\prime}$ are both split.

There are only several cases.

1. $\left(G, G^{\prime}\right)=\left(\operatorname{GL}(n), \operatorname{GL}\left(n^{\prime}\right)\right)$ with $n \leq n^{\prime}$. We can identify (a) $W=$ Mat $_{n, n^{\prime}} \oplus$ Mat $_{n, n^{\prime}}$, (b) $(x, y)^{\star}=(y,-x)$ for $(x, y) \in W, \quad\left(\right.$ c) $M(x, y)=x y^{\top} \in \mathfrak{g l}(n)$ and (d) $M(x, y)=$ $y^{\top} x \in \mathfrak{g l}\left(n^{\prime}\right)$. We set

$$
\mathscr{A}=\left\{\begin{array}{ll}
w=\left(\left(\begin{array}{ll}
a & 0
\end{array}\right),\left(\begin{array}{ll}
b & 0
\end{array}\right)\right) \mid \begin{array}{l}
a=\operatorname{diag}\left(a_{1}, \cdots, a_{n}\right) \\
b=\operatorname{diag}\left(b_{1}, \cdots, b_{n}\right)
\end{array} \text { with } a_{i}, b_{i} \in \bar{k}
\end{array}\right\} .
$$


For any $w \in \mathscr{A}, M(w)=a b$ and $M^{\prime}(w)=\left(\begin{array}{cc}a b & 0 \\ 0 & 0\end{array}\right)$.

2. $\left(G, G^{\prime}\right)=\left(\operatorname{Sp}(2 n), \mathrm{O}\left(2 n^{\prime}+1\right)\right)$ with $n \leq n^{\prime}$. We can choose suitable bases so that $V=\bar{k}^{2 n}$ and $V^{\prime}=\bar{k}^{2 n^{\prime}+1}$ such that $\left\langle v_{1}, v_{2}\right\rangle_{V}=v_{1}^{\top} J v_{2}$ and $\left\langle v_{1}^{\prime}, v_{2}^{\prime}\right\rangle_{V^{\prime}}=v_{1}^{\prime \top} J^{\prime} v_{2}^{\prime}$ where

$$
J=\left(\begin{array}{cc}
0 & I_{n} \\
-I_{n} & 0
\end{array}\right) \quad \text { and } \quad J^{\prime}=\left(\begin{array}{ccc}
0 & 0 & I_{n} \\
0 & I_{2 n^{\prime}-2 n+1} & 0 \\
I_{n} & 0 & 0
\end{array}\right)
$$

Now we can identify

(a) $W=M_{2 n^{\prime}+1,2 n}$,

(b) $w^{\star}=J^{-1} w^{\top} J^{\prime}$,

(c) $M(w)=w^{\star} w$ and

(d) $M^{\prime}(w)=w w^{\star}$. We consider

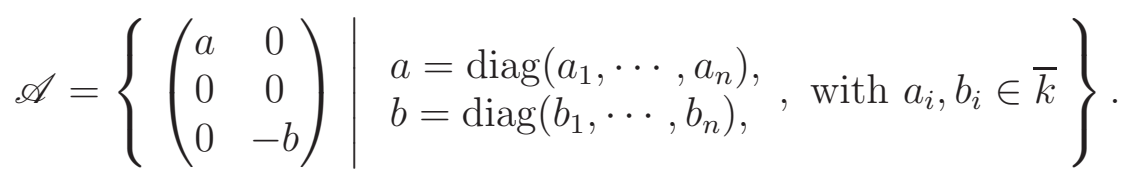

For any $w \in \mathscr{A}, M(w)=\left(\begin{array}{cc}a b & 0 \\ 0 & -a b\end{array}\right)$ and $M^{\prime}(w)=\left(\begin{array}{ccc}a b & 0 & 0 \\ 0 & 0 & 0 \\ 0 & 0 & -a b\end{array}\right)$.

3. We leave the all other cases where $\left(G, G^{\prime}\right)=\left(\operatorname{Sp}(2 n), \mathrm{O}\left(2 n^{\prime}+2\right)\right),\left(\mathrm{O}(2 n), \operatorname{Sp}\left(2 n^{\prime}\right)\right)$ or $\left(\mathrm{O}(2 n+1), \operatorname{Sp}\left(2 n^{\prime}\right)\right)$ where $n \leq n^{\prime}$ to the reader. The formulas are similar to 2 .

B.2. Proof of Lemma 8.1.1. We translate everything to the left hand side of (9) and

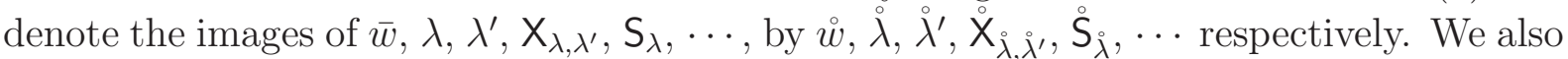
transport implicitly the Galois actions. Then $\lambda^{\circ}$ and $\dot{\lambda}^{\prime}$ are regular semisimple elements. It is enough to prove the statements for $\dot{\lambda}^{\circ}$ and $\dot{\lambda}^{\prime}$.

(i) First we assume that $\stackrel{\lambda}{\lambda} \in \operatorname{Hom}_{\mathfrak{f}_{D(E)}}(\mathrm{V}, \mathrm{V})$ is full rank. In this case $\stackrel{\circ}{w} \in \operatorname{Hom}_{\mathfrak{f}_{D(E)}}\left(\mathrm{V}, \mathrm{V}^{\prime}\right)$ is full rank too. By Witt's theorem, $\dot{M}^{\prime-1}\left(\stackrel{\prime}{\lambda}^{\prime}\right)$ is a single free $\stackrel{\circ}{\mathrm{G}}$-orbit. Let $\stackrel{\circ}{w}^{\prime} \in \dot{X}_{\dot{\lambda}, \dot{\lambda}^{\prime}}$.

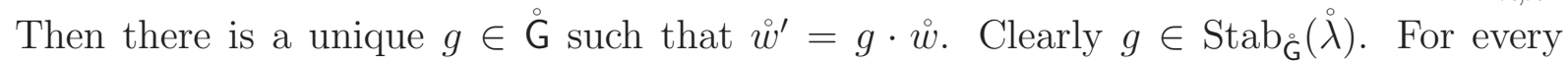
$\sigma \in \operatorname{Gal}(E / k)$,

$$
g \cdot \stackrel{\circ}{w}=\stackrel{\circ}{w}^{\prime}=\sigma\left(\stackrel{\leftrightarrow}{w}^{\prime}\right)=\sigma(g) \cdot \sigma(\stackrel{w}{w})=\sigma(g) \cdot \stackrel{\circ}{w} .
$$

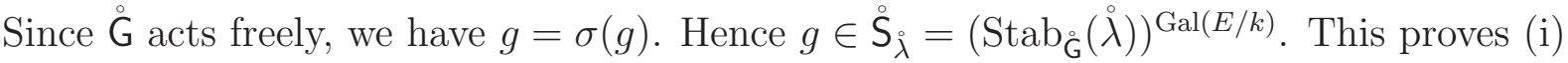
in these cases.

Now we suppose that $\stackrel{i}{\lambda}$ is not full rank i.e. Case (E). This only occurs for unitary groups of equal rank. We refer to the Appendix B.1 for the notation. In this case, $\stackrel{\mathfrak{W}}{=}=M_{n n}(\overline{\mathfrak{f}}) \oplus M_{n n}(\overline{\mathfrak{f}})$ are two copies of $n$ by $n$ matrices and $\stackrel{\lambda}{\lambda}$ is of rank $n-1$. There is an element in $\operatorname{Gal}(E / k)$ exchanging the two components of $\stackrel{\circ}{w}=(A, B)$, hence $A$ and $B$ have the same rank $n-1$. The group $\mathrm{G}$ is a general linear group. Let $\mathrm{SL}$ be the special linear group in $\stackrel{\circ}{\mathrm{G}}$. Let $\stackrel{\circ}{w}^{\prime} \in \dot{\mathrm{X}}_{\dot{\lambda}, \dot{\lambda}^{\prime}}$. Let $\mathrm{S}_{\mathrm{L}_{\grave{\lambda}}}:=\operatorname{Stab}_{\mathrm{SL}}(\stackrel{\circ}{\lambda})$. It is straightforward to check that $\stackrel{\circ}{w}^{\prime}$ and $\stackrel{\circ}{w}$ are in the same $S^{\circ} L_{\dot{\lambda}}$-orbit on which $S^{\circ} L_{\dot{\lambda}}$ acts freely. Let $g \in S^{\circ} L_{\dot{\lambda}}$ such that $\stackrel{\circ}{w}^{\prime}=g \cdot \stackrel{\circ}{w}$. Again by (21), $g$ is Galois invariant, i.e. $g \in \stackrel{\circ}{\grave{S}}_{\dot{\lambda}}$. This proves (i).

(ii) Let $w \in \mathscr{B}_{-\frac{1}{2 m}}$ be a lift of $\bar{w}$. Without loss of generality, we may assume that $w$ is of full rank. Then $\Gamma \stackrel{2 m}{=} M(w)$ and $\Gamma^{\prime}=M^{\prime}(w)$ are lifts of $\lambda$ and $\lambda^{\prime}$ respectively. Let $H_{\Gamma}$ (resp. $\left.H_{\Gamma^{\prime}}^{\prime}\right)$ be the stabilizer of $\Gamma$ (resp. $\Gamma^{\prime}$ ) in $G\left(\right.$ resp. $\left.G^{\prime}\right)$. We recall that $H_{\Gamma}$ is anisotropic so $\mathcal{B}\left(H_{\Gamma}\right)=\{x\}$ as shown in the proof of Proposition 2.3.2. This implies that $H_{\Gamma} \subseteq G_{\mathscr{L}}$ and $H_{\Gamma^{\prime}}^{\prime} \subseteq G_{\mathscr{L}^{\prime}}^{\prime}$. Using the same argument and Witt's theorem as in (i), for every $g^{\prime} \in H_{\Gamma^{\prime}}^{\prime}$ there is a unique $g \in H_{\Gamma}$ such that $g^{\prime} w=g^{-1} w$. The map $\tilde{\alpha}: H_{\Gamma^{\prime}}^{\prime} \rightarrow H_{\Gamma}$ given by $g^{\prime} \mapsto g$ 
is a surjective homomorphism. Note that $H_{\Gamma}$ (resp. $\left.H_{\Gamma^{\prime}}^{\prime}\right)$ surjects onto $\mathrm{S}_{\lambda}$ (resp. $\mathrm{S}_{\lambda^{\prime}}^{\prime}$ ) since $\Gamma$ (resp. $\Gamma^{\prime}$ ) is a good element (c.f. [13, Corollary 2.3.5 and Lemma 2.3.6]). Then $\tilde{\alpha}$ induces a homomorphism $\alpha: \mathrm{S}_{\lambda^{\prime}}^{\prime} \rightarrow \mathrm{S}_{\lambda}$.

(iii) This follows from the proofs of (i) and (ii).

(iv) Note that $\operatorname{Im} \stackrel{\circ}{*}^{\star}=\operatorname{Im} \stackrel{\lambda}{\mathrm{\lambda}} \mathrm{V}$. Let $g \in \dot{\mathrm{S}}_{\dot{\lambda}}$. Then $g \in \stackrel{\circ}{\mathrm{S}}_{\mathfrak{w}}$ if and only if $\left.g\right|_{\operatorname{Im} \mathfrak{w}^{*}}=$ id

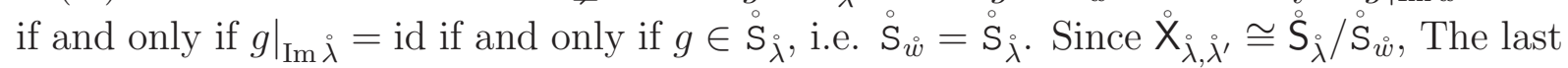
assertion is clear.

\section{Appendix C. Lattice model and splitting}

C.1. Let $\operatorname{Sp}(W)$ be a symplectic group of a symplectic space $W$. Proposition 3.3 .1 follows from Lemma C.1.1 below. One may compare the lemma with [24, §4.1] and [20].

Lemma C.1.1. Let $A_{1}$ and $A_{2}$ be two self-dual lattices in $W$. For $i=1,2$, let $\omega_{A_{i}}: \operatorname{Sp}(W) \rightarrow$ $\operatorname{Mp}(W)$ be the section defined by the lattice model $\mathscr{S}\left(A_{i}\right)$ as in (4). Then

$$
\omega_{A_{1}}(g)=\omega_{A_{2}}(g) \quad \forall g \in \operatorname{Sp}_{A_{1}} \cap \operatorname{Sp}_{A_{2}} \text {. }
$$

Proof. We have an intertwining operator $\Xi: \mathscr{S}\left(A_{1}\right) \rightarrow \mathscr{S}\left(A_{2}\right)$ given by $(\Xi f)(w)=$ $\int_{A_{2}} \psi\left(\frac{1}{2}\langle a, w\rangle\right) f(w+a) \mathrm{d} a$ between the two lattice models. This intertwining operator is unique up to scalar.

Let $g \in \operatorname{Sp}_{A_{1}} \cap \mathrm{Sp}_{A_{2}}$. Since $g: A_{2} \rightarrow A_{2}$ is measure preserving,

$$
\begin{aligned}
\left(\left(\omega_{A_{2}}(g) \circ \Xi f\right)(w)\right. & =\int_{A_{2}} \psi\left(\frac{1}{2}\left\langle a, g^{-1} w\right\rangle\right) f\left(g^{-1} w+a\right) \mathrm{d} a \\
& =\int_{A_{2}} \psi\left(\frac{1}{2}\langle g a, w\rangle\right) f\left(g^{-1} w+a\right) \mathrm{d} a \\
& =\int_{A_{2}} \psi\left(\frac{1}{2}\langle a, w\rangle\right) f\left(g^{-1} w+g^{-1} a\right) \mathrm{d} a \\
& =\left(\Xi \circ \omega_{A_{1}}(g) f\right)(w) .
\end{aligned}
$$

This proves the lemma.

C.2. Let $x \in \mathcal{B}(\mathbf{G}, k)$. We pick any $x^{\prime} \in \mathcal{B}\left(\mathbf{G}^{\prime}, k\right)$ and let $\mathscr{L}$ and $\mathscr{L}^{\prime}$ be the lattice functions corresponding to $x$ and $x^{\prime}$ respectively. Let $\mathscr{B}=\mathscr{L} \otimes \mathscr{L}^{\prime}$ be the tensor product lattice function and $A$ be any self-dual lattice such that $\mathscr{B}_{0^{+}} \subseteq A \subseteq \mathscr{B}_{0}$. We have $G_{x, 0^{+}}$ stabilizes $A$, i.e. $G_{x, 0^{+}} \subseteq \operatorname{Sp}_{A}$ (see also [22, §3.3.2]). As a corollary of Lemma C.1.1, the lattice models give a canonical splitting

$$
\omega_{+}: \bigcup_{x \in \mathcal{B}(\mathbf{G}, k)} G_{x, 0^{+}} \longrightarrow \widetilde{G} .
$$

\section{REFERENCES}

[1] J. Adams, L-Functoriality for Dual Pairs, Asterisque 171-172 (1989), 85-129.

[2] J. D. Adler and A. Roche, An intertwining result for p-adic groups, Canad. J. Math 52 (200), no. 3, 449-467.

[3] P. Broussous and S. Stevens, Buildings of classical groups and centralizers of Lie algebra elements, Journal of Lie Theory 19 (2009), 55-78.

[4] F. Bruhat and J. Tits, Schémas en groupes et immeubles des groupes classiques sur un corps local, Bull. Math. Soc. France 112 (1984), 259-301.

[5] _ Schémas en groupes et immeubles des groupes classiques sur un corps local, II. Groupes unitaires, Bull. Math. Soc. France 115 (1987), 141-195.

[6] W. T. Gan and S. Takeda, A proof of the Howe duality conjecture, To appear in Journal of AMS, arXiv:1407.1995 (2014). 
[7] R. Goodman and N. R Wallach, Symmetry, representations, and invariants, GTM, vol. 255, Springer, 2009.

[8] G. Prasad and J.-K. Yu, On finite group actions on reductive groups and buildings, Inventiones mathematicae 147 (2202), no. 3, 545-560.

[9] B. Gross, P. Levy, M. Reeder, and J.-K. Yu, Gradings of positive rank on simple Lie algebras, Transformation Groups 17 (2012), no. 4, 1123-1190.

[10] R. Howe, - -series and invariant theory, Proc. Symp. Pure Math. 33 (1979), Part 1, 275-285.

[11] _ Perspectives on invariant theory: Schur duality, multiplicity-free actions and beyond, Piatetski-Shapiro, Ilya (ed.) et al., The Schur lectures (1992). Ramat-Gan: Bar-Ilan University, Isr. Math. Conf. Proc. 8 (1995), 1-182.

[12] J.-L. Kim, Supercuspidal Representations: An Exhaustion Theorem, Journal of the American Mathematical Society 20 (2007), no. 2, 273-320.

[13] J.-L. Kim and F. Murnaghan, Character expansions and unrefined minimal K-types, American Journal of Mathematics 125 (2003), no. 6, 1199-1234.

[14] B. Lemaire, Comparison of lattice filtrations and Moy-Prasad filtrations for classical groups, Journal of Lie Theory 19 (2008), no. 1, 029-054.

[15] P. Levy, Vinbergs $\theta$-groups in positive characteristic and KostantWeierstrass slices, Transformation Groups 14 (2009), no. 2, 417-461.

[16] G. J. McNinch, Levi Factors of the Special Fiber of a Parahoric Group Scheme and Tame Ramification, Algebras and Representation Theory 17 (2014), no. 2, 469-479.

[17] C. Møglin, M. F. Vigneras, and J.-L. Waldspurger, Correspondances de Howe sur un corps p-adique, Lecture Notes in Mathematics, vol. 1291, Springer Verlag, Berlin, 1987.

[18] C. Moen, The dual pair (U(1), U(1)) over a p-adic field., Pacific J. Math. 158 (1993), no. 2, 365-386.

[19] A Moy and G Prasad, Unrefined minimal K-types for p-adic groups, Inventiones Math. 116 (1994), 393-408.

[20] S.-Y. Pan, Splittings of the metaplectic covers of some reductive dual pairs, Pacific J. Math. 199 (2001), no. 1, 163-226.

[21] — Depth preservation in local theta correspondence, Duke Math. J. 113 (2002), no. 3, 531-592.

[22] _ Local theta correspondence and minimal K-types of positive depth, Israel J. Math. 138 (2003), 317-352.

[23] M. Reeder and J.-K. Yu, Epipelagic representations and invariant theory, J. Amer. Math. Soc. 27 (2014), 437-477.

[24] B. Sun and C.-B. Zhu, Conservation relations for local theta correspondence, to appear J. Amer. Math. Soc. (2014).

[25] J. Tits, Reductive groups over local fields, Automorphic forms, representations and L-functions I, Amer. Math. Soc., Providence, R.I, 1979, pp. 29-69.

[26] E. B. Vinberg, The Weyl group of a graded Lie algebra, Izv. Akad. Nauk SSR 40 (1976), no. 3, 463-495.

[27] J.-L. Waldspurger, Démonstration dune conjecture de dualité de Howe dans le cas $p$-adique, $p \neq 2$ in Festschrift in honor of I. I. Piatetski-Shapiro on the occasion of his sixtieth birthday, Israel Math. Conf. Proc., 2, Weizmann, Jerusalem (1990), 267-324.

Hung Yean Loke, Department of Mathematics, National University of Singapore, Block S17, 10 Lower Kent Ridge Road, Singapore 119076

E-mail address: matlhy@nus.edu.sg

Jia-Jun Ma, Unit 408, Academic Building No.1, The Institute of Mathematical Sciences, The Chinese University of Hong Kong, Shatin, N.T., Hong Kong

E-mail address: jjma@ims.cuhk.edu.hk

Gordan Savin, Department of Mathematics University of Utah Salt Lake City, UT 84112

E-mail address: savin@math.utah.edu 